\title{
OPTIMAL RANK-BASED PROCEDURES FOR TIME SERIES ANALYSIS: TESTING AN ARMA MODEL AGAINST OTHER ARMA MODELS
}

\author{
By Marc Hallin and Madan L. Puri ${ }^{1}$ \\ Université Libre de Bruxelles and Indiana University
}

\begin{abstract}
The problem of testing a given ARMA model (in which the density of the generating white noise is unspecified) against other ARMA models is considered. A distribution-free asymptotically most powerful test, based on a generalized linear serial rank statistic, is provided against contiguous ARMA alternatives with specified coefficients. In the case when the ARMA model in the alternative has unspecified coefficients, the asymptotic sufficiency (in the sense of Le Cam) of a finite-dimensional vector of rank statistics is established. This asymptotic sufficiency is used to derive an asymptotically maximin most powerful test, based on a generalized quadratic serial rank statistic. The asymptotically maximin optimal test statistic can be interpreted as a rank-based, weighted version of the classical Box-Pierce portmanteau statistic, to which it reduces, in some particular problems, asymptotically and under Gaussian assumptions.
\end{abstract}

\section{Introduction.}

1.1. Rank tests for time series analysis. Time series analysis is certainly one of the areas in statistics whose development has been most impressive during the past two decades, and its importance for practical applications, ranging from economics to engineering has been widely recognized. However in spite of the growing interest in the subject and in spite of the fact that the need for robust or rank-based procedures has been emphasized by many authors, not much has been done to introduce such procedures (especially the rank-based ones) in the time series context.

This does not mean, of course, that no attempt was ever made to use rank-based methods beyond the classical problems involving i.i.d. (independent and identically distributed) observations. A very comprehensive bibliography on rank tests for non-i.i.d. observations can be found in a paper entitled "Nonparametric testing for time series analysis: A bibliography," by Dufour, Lepage and Zeidan (1982). This title however is somewhat misleading inasmuch as most of the papers mentioned on rank-based methods deal with such problems as testing for randomness (i.i.d. observations) against trend alternatives (trend in location, in dispersion, ordered alternatives,...) under which observations are nonidentically but still independently distributed, whereas time series analysis is typically

Received May 1985; revised May 1987.

${ }^{1}$ Research supported by Office of Naval Research Contract N00014-85-K-0648.

AMS 1980 subject classifications. Primary 62M10, 62G10.

Key words and phrases. ARMA models, linear serial rank statistics, quadratic rank statistics, rank portmanteau statistics, asymptotic sufficiency, asymptotically most powerful tests, asymptotically maximin most powerful tests. 
concerned with nonindependent observed data and their serial dependence structure. Thus, it appears from this bibliography along with more recent literature that, apart from some scattered and very piecemeal results on the problem of testing for white noise against serial dependence [cf. Bhattacharyya (1984) or Hallin, Ingenbleek and Puri (1985) for a review], the subject of rank tests in time series analysis remains largely unexplored.

A first step toward a systematic treatment of this subject was taken in two papers by Hallin, Ingenbleek and Puri (1985 and 1987), where locally asymptotically optimal and locally maximin-optimal rank tests (in the Pitman-Noether sense) were derived for the problem of testing randomness against alternatives of ARMA dependence. Two classes of serial rank statistics were introduced for that purpose.

The first one is the class of linear serial rank statistics, of order $p$, of the form

$$
S^{(n)}=(n-p)^{-1} \sum_{t=p+1}^{n} a^{(n)}\left(R_{t}^{(n)}, R_{t-1}^{(n)}, \ldots, R_{t-p}^{(n)}\right)
$$

where $R_{t}^{(n)}$ denotes the rank of the observation $X_{t}^{(n)}$ in an observed series $\mathbf{X}^{(n)}=\left(X_{1}^{(n)}, \ldots, X_{n}^{(n)}\right)$ of length $n$, and $a^{(n)}(\cdots)$ is some score function. Of special interest is the particular linear serial rank statistic, namely, the rank autocorrelation $r_{i ; f}^{(n)}$ [see (2.5) for definition] of order $i$ associated with density $f(\cdot)$ which enjoys most of the asymptotic properties of the usual sample autocorrelation $r_{i}^{(n)}$ for Gaussian series [cf. Hallin, Ingenbleek and Puri (1987) and Hallin and Puri (1987)].

The second class of rank statistics considered is that of quadratic serial rank statistics, which are quadratic forms of linear serial rank statistics [cf. Hallin, Ingenbleek and Puri (1987)]. Of particular interest are the rank portmanteau statistics $n \sum_{i=1}^{\kappa}\left(r_{i ; f}^{(n)}\right)^{2}$ which provide a rank version of Box and Pierce's (1970) classical portmanteau statistic $n \sum_{i=1}^{k}\left(r_{i}^{(n)}\right)^{2}$.

1.2. Testing randomness against ARMA alternatives. Consider the sequence of $\operatorname{ARMA}(p, q)$ models

$$
X_{t}-n^{-1 / 2} \sum_{i=1}^{p} a_{i} X_{t-i}=\varepsilon_{t}+n^{-1 / 2} \sum_{i=1}^{q} b_{i} \varepsilon_{t-i}, \quad t \in \mathbb{Z},
$$

where $\left\{\varepsilon_{t}\right\}$ is an independent white noise, i.e., a family of i.i.d. random variables with mean zero, unspecified variance $\sigma^{2}$ and density function $f(\cdot)$ ( $f$ has to satisfy some mild technical assumptions; cf. Section 2). Let $a_{i}=0, i>p$ and $b_{i}=0, i>q$; put $\mathbf{a}=\left(a_{1}, \ldots, a_{\max (p, q)}\right)$ and $\mathbf{b}=\left(b_{1}, \ldots, b_{\max (p, q)}\right)$ and denote by $\|\cdot\|$ the usual Euclidean norm. The vectors $\mathbf{a}$ and $\mathbf{b}$ and the density $f$ determine what we call here a specified ARMA alternative $K^{(n)}(\mathbf{a}, \mathbf{b} ; f)$. Denote by $H_{0}^{(n)}$ the null hypothesis of randomness, under which $\mathbf{X}^{(n)}$ is a vector of i.i.d. random variables with unspecified density function. Then an asymptotically most powerful $\alpha$-level test $\varphi_{\alpha}^{(n)}(\mathbf{a}, \mathbf{b} ; f)$ (among all tests of given level $\alpha$ ) for 
testing randomness against $K^{(n)}(\mathbf{a}, \mathbf{b} ; f)$ is given by

$$
\begin{gathered}
\varphi_{\alpha}^{(n) *}(\mathbf{a}, \mathbf{b} ; f)=1 \\
\text { if } \sum_{i=1}^{\max (p, q)}(n-i)^{1 / 2}\left(a_{i}+b_{i}\right) r_{i ; f}^{(n)}>\|\mathbf{a}+\mathbf{b}\| k_{1-\alpha},
\end{gathered}
$$

where $k_{1-\alpha}$ denotes the $(1-\alpha)$-quantile of the standard normal distribution. In the Gaussian case [assuming $E\left(X_{t}\right)=0$ ], (1.3) can also be expressed, under asymptotically equivalent "parametric" form, in terms of the sample autocorrelations $r_{i}^{(n)}=\sum_{t=i+1}^{n} X_{t}^{(n)} X_{t-i}^{(n)} / \sum_{t=1}^{n}\left(X_{t}^{(n)}\right)^{2}$, as

$$
\begin{gathered}
\varphi_{\alpha}^{(n) *}(\mathbf{a}, \mathbf{b} ; f)=1 \\
\text { if } \sum_{i=1}^{\max (p, q)}(n-i)^{1 / 2}\left(a_{i}+b_{i}\right) r_{i}^{(n)}>\|\mathbf{a}+\mathbf{b}\| k_{1-\alpha} .
\end{gathered}
$$

These results follow from Proposition 3.2 in Hallin, Ingenbleek and Puri (1985); they can also be obtained as particular cases of Propositions 2.4 and 4.3 by letting $p_{1}=q_{1}=0$ [hence $\left.A(L)=B(L)=1\right]$ in (3.2) and (4.2).

Of primary importance from the practical viewpoint is the development of the theory when the coefficients $\mathbf{a}$ and $\mathbf{b}$ in the ARMA model (1.2) are unspecified. We thus consider the alternative $K_{f}^{(n)}(m)$ consisting of all $\operatorname{ARMA}(p, q)$ dependencies (1.2) such that $\max (p, q) \leq m: K_{f}^{(n)}(m)=U\left\{K^{(n)}(\mathbf{a}, \mathbf{b} ; f) \mid \mathbf{a}+\mathbf{b} \in\right.$ $\left.\mathbb{R}^{m}\right\}$. Also, denote by $K_{f}^{(n)}(m \mid d)$, where $d \in \mathbb{R}^{+}$, the family of all ARMA dependencies in $K_{f}^{(n)}(m)$ such that $\|\mathbf{a}+\mathbf{b}\| \geq d$.

Then an asymptotically maximin most powerful $\alpha$-level test $\psi_{\alpha}^{(n) *}(m ; f)$ (among all tests of given level $\alpha$ ) for randomness against any alternative $K_{f}^{(n)}(m \mid d), d \in \mathbb{R}^{+}$, can be based on the rank portmanteau statistic of order $m$,

$$
\psi_{\alpha}^{(n) *}(m ; f)=1 \quad \text { if } \quad \sum_{i=1}^{m}(n-i)\left(r_{i ; f}^{(n)}\right)^{2}>\chi_{m ; 1-\alpha}^{2},
$$

where $\chi_{m ; 1-\alpha}^{2}$ denotes the $(1-\alpha)$-quantile of the $\chi^{2}$ distribution with $m$ degrees of freedom. In the Gaussian case, (1.5) can also be expressed, under asymptotically equivalent "parametric" form, in terms of the classical Box-Pierce portmanteau statistic

$$
\psi_{\alpha}^{(n) *}(m ; f)=1 \quad \text { if } \quad \sum_{i=1}^{m}(n-i)\left(r_{i}^{(n)}\right)^{2}>\chi_{m ; 1-\alpha}^{2} .
$$

These latter results follow, as particular cases, from Proposition 4.3.

1.3. Testing an ARMA model against another ARMA model. Testing for white noise (with unspecified density function) against ARMA dependence is certainly a very important problem in time series analysis, mainly because the nonrejection of a null hypothesis of randomness implies that complicated time series analysis procedures can be safely replaced with much simpler and more traditional devices such as the usual linear model methods. As pointed out by 
Dufour and Roy (1985), tests for randomness can also be used to check several important economic hypotheses, such as market efficiency, rational expectations, life cycle-permanent income hypotheses, etc.

The problem of testing a given ARMA model (with unspecified density function) against other ARMA models is, however, even more important because of its implications in the various identification and validation steps that are part of any time series model-building procedure.

Denote by $H^{(n)}(\mathrm{A}, \mathrm{B} ; \cdot)$ the null hypothesis under which the observed series $\mathbf{X}^{(n)}$ is generated by the $\operatorname{ARMA}\left(p_{1}, q_{1}\right)$ model

$$
\begin{aligned}
A(L) X_{t} & =X_{t}-A_{1} X_{t-1}-\cdots-A_{p_{1}} X_{t-p_{1}} \\
& =\varepsilon_{t}+B_{1} \varepsilon_{t-1}+\cdots+B_{q_{1}} \varepsilon_{t-q_{1}}=B(L) \varepsilon_{t}, \quad t \in \mathbb{Z},
\end{aligned}
$$

where $\left\{\varepsilon_{t}\right\}$ is a white noise process with unspecified density function $[L$ denotes the lag operator and $A(L)=1-A_{1} L-\cdots-A_{p_{1}} L^{p_{1}}, B(L)=1+$ $B_{1} L+\cdots+B_{q_{1}} L^{q_{1}}$ are linear difference operators]. Consider the filtered process $\left\{Z_{t}=[A(L)] /[B(L)] X_{t}\right\}$ and the filtered series $\mathbf{Z}^{(n)}=\left(Z_{1}^{(n)}, \ldots, Z_{n}^{(n)}\right)$ associated with the observed series $\mathbf{X}^{(n)}$ (at this stage, we disregard the "starting values" problem involved in the definition of $\mathbf{Z}^{(n)}$; as shown in Appendix 2, these starting values do not affect asymptotic results). Obviously, $\mathbf{X}^{(n)}$ is an $\operatorname{ARMA}\left(p_{1}, q_{1}\right)$ series generated by (1.7) iff $\mathbf{Z}^{(n)}$ is a white noise series. At first sight, the problem of testing $H^{(n)}(\mathbf{A}, \mathbf{B} ; \cdot)$ for the original series $\mathbf{X}^{(n)}$ thus reduces to the previously solved problem of testing for white noise $\left(H_{0}^{(n)}\right.$ for the filtered series $\mathbf{Z}^{(n)}$ ). This is true, actually, as far as the null hypothesis is considered; let us now turn to the alternative.

The alternative hypotheses of interest here are those under which $\mathbf{X}^{(n)}$ is an observed series from some ARMA model $\alpha(L) X_{t}=\beta(L) \varepsilon_{t}$ distinct from (1.7). In order to investigate locally optimal procedures, we shall consider sequences of alternatives that are contiguous to the null hypotheses (see Section 2.2). Let

$$
\begin{aligned}
\alpha^{(n)}(L) X_{t} & =X_{t}-\alpha_{1}^{(n)} X_{t-1}-\cdots-\alpha_{p_{2}}^{(n)} X_{t-p_{2}} \\
& =\varepsilon_{t}+\beta_{1}^{(n)} \varepsilon_{t-1}+\cdots+\beta_{q_{2}}^{(n)} \varepsilon_{t-q_{2}}=\beta^{(n)}(L) \varepsilon_{t}, \quad t \in \mathbb{Z},
\end{aligned}
$$

be a sequence of $\operatorname{ARMA}\left(p_{2}, q_{2}\right)$ models, with $p_{2} \geq p_{1}, q_{2} \geq q_{1}$ and

$$
\begin{aligned}
\alpha_{i}^{(n)} & = \begin{cases}A_{i}+n^{-1 / 2} \gamma_{i}, & 1 \leq i \leq p_{1}, \\
n^{-1 / 2} \gamma_{i}, & p_{1}<i \leq p_{2},\end{cases} \\
\beta_{i}^{(n)} & = \begin{cases}B_{i}+n^{-1 / 2} \delta_{i}, & 1 \leq i \leq q_{1}, \\
n^{-1 / 2} \delta_{i}, & q_{1}<i \leq q_{2} .\end{cases}
\end{aligned}
$$

Denote by $\gamma$ and $\delta$ the vectors $\left(\gamma_{1}, \ldots, \gamma_{p_{2}}\right)$ and $\left(\delta_{1}, \ldots, \delta_{q_{2}}\right)$, respectively, and by $K^{(n)}(\mathbf{A}, \mathbf{B} ; \gamma, \delta ; f)$ the sequence of $\operatorname{ARMA}\left(p_{2}, q_{2}\right)$ alternatives (1.8) corresponding to specified $\gamma, \delta$ and $f$. If $\mathbf{X}^{(n)}$ is an observed series from (1.8), it is easy to see that the filtered series $\mathbf{Z}^{(n)}$ is generated by the model

$$
\alpha^{(n)}(L) B(L) Z_{t}=A(L) \beta^{(n)}(L) \varepsilon_{t}, \quad t \in \mathbb{Z},
$$


an $\operatorname{ARMA}(p, q)$ model with $p_{2}-p_{1} \leq p \leq p_{2}+q_{1}$ and $q_{2}-q_{1} \leq q \leq p_{1}+q_{2}$. The model (1.10) is not a model of the type (1.2) considered in Section 1.2, except for the very particular case where $\alpha^{(n)}(L)$ and $\beta^{(n)}(L)$ are of the form

$$
\begin{aligned}
& \alpha^{(n)}(L)=A(L)\left(1-n^{-1 / 2}\left(a_{1} L+\cdots+a_{p} L^{p}\right)\right), \\
& \beta^{(n)}(L)=B(L)\left(1+n^{-1 / 2}\left(b_{1} L+\cdots+b_{q} L^{q}\right)\right),
\end{aligned}
$$

yielding, for the filtered series, the ARMA $\left(p=p_{2}-p_{1}, q=q_{2}-q_{1}\right)$ model

$$
Z_{t}-n^{-1 / 2}\left(a_{1} Z_{t-1}+\cdots+a_{p} Z_{t-p}\right)=\varepsilon_{t}+n^{-1 / 2}\left(b_{1} \varepsilon_{t-1}+\cdots+b_{q} \varepsilon_{t-q}\right) \text {. }
$$

Consequently, the problem of testing an ARMA model (for the original series $\left.\mathbf{X}^{(n)}\right)$ against another one [viz. testing $H^{(n)}(\mathbf{A}, \mathbf{B} ; \cdot)$ against $K^{(n)}(\mathbf{A}, \mathbf{B} ; \gamma, \delta ; f)$ ] does not, in general, reduce to the previously studied problem of testing for white noise (for the filtered series $\mathbf{Z}^{(n)}$ ) against ARMA dependence [viz. testing $H_{0}^{(n)}$ against $\left.K^{(n)}(\mathbf{a}, \mathbf{b} ; f)\right]$, and the results of Hallin, Ingenbleek and Puri $(1985,1987)$ are no longer applicable to this new problem. The optimal tests indeed will be shown to depend, in a very crucial way, on the tested model (1.7).

1.4. Outline of the paper. We first consider, in Section 2, the problem of testing for randomness against contiguous specified alternatives of general linear dependence. The asymptotic distribution of the log-likelihood ratio for this problem is derived in Proposition 2.2, and an asymptotically most powerful rank test is provided in Proposition 2.4. These results are then used in Section 3, where we show that the model (1.10) describing the behaviour of the filtered series $\mathbf{Z}^{(n)}$ under the alternative, in the problem of testing an ARMA model against some other ARMA model, is a particular case of the general linear model considered in Section 2. An asymptotically most powerful statistic for testing a given ARMA model against another one is given in Section 3.2, where we also establish the asymptotic sufficiency [in the sense of Le Cam (1960) and Hájek and Šidák (1967)] of a vector of $\max \left(p_{1}+q_{2}, p_{2}+q_{1}\right)$ rank statistics [see (1.8) and (1.9) for a definition of $p_{1}, p_{2}, q_{1}$ and $q_{2}$ ]. These statistics involve the $f$-rank autocorrelations (of all available orders) and an arbitrary fundamental system of solutions of the homogeneous difference equation $A(L) B(L) \Psi_{t}=0$, $t \in \mathbb{Z}$, defined by the ARMA model (1.7) to be tested. The asymptotic normal distribution of this sufficient statistic is provided in Proposition 3.3.

It follows (cf. Section 3.2) from the definition of asymptotic sufficiency that, under certain conditions, asymptotically maximin most powerful tests can be based on asymptotically sufficient statistics. We therefore introduce, in Section 4.1, a generalized quadratic serial rank statistic extending the concept introduced in Hallin, Ingenbleek and Puri (1987). The definition of this quadratic statistic, although involving an arbitrary fundamental system of solutions of the homogeneous equation $A(L) B(L) \Psi_{t}=0, t \in \mathbb{Z}$, is shown not to depend on the particular system adopted (Proposition 4.1); examples are provided.

The asymptotic $\chi^{2}$ (under the null hypotheses) and noncentral $\chi^{2}$ (under the alternative) distributions of this statistic are obtained in Proposition 4.2, and the asymptotic maximin optimality of the corresponding test is proved in Proposi- 
tion 4.3. The form of the maximin-optimal statistic is discussed in some detail in Section 4.2. Section 5 provides some results on the asymptotic relative efficiencies of our rank tests with respect to some classical parametric tests (such as the classical Box-Pierce or Durbin-Watson tests).

The ranks used throughout the paper are the ranks of residuals whose derivation may require the inversion of a moving-average operator. Such residuals in this case cannot be obtained from a finite series of observations, and approximate residuals have to be substituted for the exact ones. Appendix 2 shows that this use of approximate residuals has no influence upon the results of the paper. Appendix 1 provides a brief overview of some results on linear difference equations which are used continuously throughout the paper. Appendix 3 concentrates on the proofs of the propositions.

\section{General linear processes.}

2.1. The general linear alternative. Denote by $\left\{\varepsilon_{t} ; t \in \mathbb{Z}\right\}$ a discrete-time stationary white noise, i.e., a sequence of independent and identically distributed real-valued random variables with mean $E\left(\varepsilon_{t}\right)=0$ and (unspecified) variance $E\left(\varepsilon_{t}^{2}\right)=\sigma^{2}$. Assume that these random variables have a density function $f(x)$ (with respect to the Lebesgue measure) and that the following technical conditions are satisfied:

1. $\varepsilon_{t}$ has finite moments up to the sixth order.

2. $f(x)$ is (a.e.) derivable [denote by $f^{\prime}(x)$ its derivative], absolutely continuous on finite intervals [see Hájek and Šidák (1967), page 15] and satisfies $E\left[\left|f^{\prime}\left(\varepsilon_{t}\right) / f\left(\varepsilon_{t}\right)\right|^{2+\delta}\right]<\infty$ for some $\delta>0$ [this implies that $f(x)$ has finite Fisher information $\left.I(f): 0<I(f)=E\left[\left(f^{\prime}\left(\varepsilon_{t}\right) / f\left(\varepsilon_{t}\right)\right)^{2}\right]<\infty\right]$.

3. Denote by $F(x)$ the cumulative distribution function associated with $f(x)$ and let $F^{-1}(u)=\inf \{x \mid F(x) \geq u\}, \quad 0<u<1$. Define $\phi(x)$ as $-f^{\prime}\left(F^{-1}(F(x))\right) / f\left(F^{-1}(F(x))\right)$. Assume that $\phi(x)$ is a.e. derivable and that its derivative $\phi^{\prime}(x)$ is (a.e.) Lipschitzian and square integrable: $\left|\phi^{\prime}(x)-\phi^{\prime}(y)\right|<$ $K|x-y|\left(K, K^{\prime}\right.$ and $K^{\prime \prime}$ will be used throughout the paper to denote constants-not necessarily the same), and $\int_{0}^{1} \phi^{\prime 2}\left(F^{-1}(u)\right) d u<\infty$. Notice that $\phi(x)$ is a.e. equal to $-f^{\prime}(x) / f(x)$.

4. $f$ is strongly unimodal [cf. Hájek and Šidák (1967), page 15].

Let $\mathbf{a}=\left(a_{1}, a_{2}, \ldots\right)$ and $\mathbf{b}=\left(b_{1}, b_{2}, \ldots\right)$ be two sequences of real coefficients such that $\sum_{i=1}^{\infty}\left|a_{i}\right|<\infty$ and $\sum_{i=1}^{\infty}\left|b_{i}\right|<\infty$. This implies that $\mathbf{a}$ and $\mathbf{b}$ are elements of the Hilbert space $l^{2}$ of the square-summable sequences, which ensures that, for $n$ sufficiently large (we henceforth shall assume that the following holds for $n \in \mathbb{N})$, the functions $1-n^{-1 / 2} \sum_{i=1}^{\infty} a_{i} z^{i}$ and $1+n^{-1 / 2} \sum b_{i} z^{i}$ are both analytic inside and on the unit circle, as well as their inverses. The stochastic difference equation (of "infinite order")

$$
X_{t}-n^{-1 / 2} \sum_{i=1}^{\infty} a_{i} X_{t-i}=\varepsilon_{t}+n^{-1 / 2} \sum_{i=1}^{\infty} b_{i} \varepsilon_{t-i}, \quad t \in \mathbf{Z},
$$


therefore correctly defines a unique stationary general linear process $\left\{X_{t}^{(n)}\right\}$, i.e., a process of the form

$$
X_{t}^{(n)}=\varepsilon_{t}+\sum_{i=1}^{\infty} c_{i}^{(n)} \varepsilon_{t-i}, \quad t \in \mathbb{Z},
$$

with $\sum_{i=1}^{\infty}\left(c_{i}^{(n)}\right)^{2}<\infty$ [see, e.g., Priestley (1981), Section 3.5.7]. As can be easily seen from (2.2), $\left\{X_{t}^{(n)}\right\}$ has finite sixth-order moments if $\left\{\varepsilon_{t}\right\}$ has. Notice, however, that the coefficients $c_{i}^{(n)}$ in (2.2) are generally not of the form $n^{-1 / 2} c_{i}$, so that (2.2) is not a particular case of (2.1).

Let $\mathbf{x}^{(n)}=\left(x_{1}^{(n)}, \ldots, x_{n}^{(n)}\right)$ be the value of an observed series $\mathbf{X}^{(n)}=$ $\left(X_{1}^{(n)}, \ldots, X_{n}^{(n)}\right)$ of length $n$. Denote by $K_{f}^{(n)}$ the hypothesis under which $\mathbf{X}_{n}$ is a finite realization of some general linear process $\left\{X_{t}^{(n)}\right\}$, defined by (2.1) with unspecified sequences $\mathbf{a}$ and $\mathbf{b}$ and a specified input white noise density $f(x)$ satisfying $1-3$; let $K^{(n)}(\mathbf{a}, \mathbf{b} ; f)$ be the subhypothesis obtained by specifying a and $\mathbf{b}$. Such hypotheses will be referred to as (specified or unspecified) general linear alternatives. Clearly, general linear alternatives constitute a generalization of the ARMA alternatives $K^{(n)}\left(\mathbf{a}=\left(a_{1} \cdots a_{p}\right), \mathbf{b}=\left(b_{1} \cdots b_{q}\right) ; f\right)$ previously considered in Section 1.2 (and this is why we adopt similar notations); they also include the hypotheses of the form $K^{(n)}(\mathbf{A}, \mathbf{B} ; \gamma, \delta ; f)$ (see Section 1.3) we shall be interested in when testing an ARMA model against other ARMA models-this latter statement will be made clearer in Section 3.1.

In Sections 2.2 and 2.3, we mainly establish for general linear alternatives some results (viz. contiguity and the form of asymptotically most powerful tests) that will be needed in the problem of testing an ARMA model against other ARMA models. First let us state a theorem due to Anderson [(1959); cf. Anderson (1971), Theorem 7.7.1] which will be used repeatedly.

Theorem 2.1. Let $Y^{(n)}=Y_{\kappa}^{(n)}+R_{\kappa}^{(n)}, n \in \mathbb{N}, \kappa \in \mathbb{N}$. Suppose that $R_{\kappa}^{(n)}$ converges to zero in probability, as $\kappa \rightarrow \infty$, uniformly with respect to $n$ [i.e., for any $\varepsilon^{\prime}>0, \varepsilon^{\prime \prime}>0$ there exists a $\kappa_{0}\left(\varepsilon^{\prime}, \varepsilon^{\prime \prime}\right)$ not depending on $n$ such that $P\left[\left|R_{\kappa}^{(n)}\right|>\varepsilon^{\prime}\right]<\varepsilon^{\prime \prime}$ for any $\left.\kappa>\kappa_{0}\right]$ and that the distribution functions $F_{\kappa}^{(n)}(y)=P\left[Y_{\kappa}^{(n)} \leq y\right]$ converge, as $n \rightarrow \infty$, to some distribution function $F_{\kappa}(y)$ such that $\lim _{\kappa \rightarrow \infty} F_{\kappa}(y)=F(y)$ at every continuity point of $F(y)$. Then $\lim _{n \rightarrow \infty} P\left[Y^{(n)} \leq y\right]=F(y)$ at every continuity point of $F(y)$.

2.2. Generalized linear serial rank statistics. In Hallin, Ingenbleek and Puri (1985), we established the contiguity of sequences of finite-order ARMA alternatives and the white noise hypothesis $H_{0}^{(n)}$. Proposition 2.2 extends this result to general linear hypotheses of the form $K^{(n)}(\mathbf{a}, \mathbf{b} ; f)$.

Denote by $L^{(n)}\left(\mathbf{X}^{(n)}\right)$ the likelihood ratio for testing $H_{0 ; f}^{(n)}$ (the white noise hypothesis with specified density $f$ ) against $K^{(n)}(\mathbf{a}, \mathbf{b} ; f)$. Then Proposition 2.2 is proved in Appendix 3.

Proposition 2.2. The log-likelihood ratio $\log L^{(n)}\left(\mathbf{X}^{(n)}\right)$ is asymptotically normal, with mean $-\frac{1}{2} \sum_{i=1}^{\infty}\left(a_{i}+b_{i}\right)^{2} \sigma^{2} I(f)$ and variance $\sum_{i=1}^{\infty}\left(a_{i}+b_{i}\right)^{2} \sigma^{2} I(f)$ 
( under $\left.H_{0 ; f}^{(n)}\right)$. The sequences of hypotheses $K^{(n)}(\mathbf{a}, \mathbf{b} ; f)$ and $H_{0}^{(n)}$ are therefore contiguous.

Linear serial rank statistics of order $p$ defined in (1.1) consider successive $(p+1)$-tuples of observations and consequently cannot be expected to account for serial dependencies of orders higher than $p$. This is confirmed by Proposition 2.3, which motivates the introduction of generalized linear serial rank statistics.

Consider the linear serial rank statistics $S^{(n)}$ of order $p$ given in (1.1). Let

$$
m^{(n)}=\frac{(n-p-1) !}{n !} \sum_{1 \leq i_{1} \neq \cdots \neq i_{p+1} \leq n} a_{n}\left(i_{1}, \ldots, i_{p+1}\right)
$$

be the expectation of $S^{(n)}$ under $H_{0}^{(n)}$. Assume that there exists a function $J(\cdots)$ defined over $(0,1)^{p+1}$ such that

$$
\int_{[0,1]^{p+1}}\left|J\left(v_{1}, \ldots, v_{p+1}\right)\right|^{2+\delta} d v_{1} \cdots d v_{p+1}<\infty \text { for some } \delta>0,
$$

satisfying (under $H_{0}^{(n)}$ )

$$
\lim _{n \rightarrow \infty} E\left\{\left[J\left(F\left(X_{1}^{(n)}\right), \ldots, F\left(X_{p+1}^{(n)}\right)\right)-a_{n}\left(R_{1}^{(n)}, \ldots, R_{p+1}^{(n)}\right)\right]^{2}\right\}=0 .
$$

$J(\cdots)$ is called a score-generating function associated with $S^{(n)}$. We furthermore may assume without loss of generality [cf. Hallin, Ingenbleek and Puri 1987)] that

$$
\int_{[0,1]^{p+1}} J\left(v_{1}, \ldots, v_{p+1}\right) \prod_{j \neq i} d v_{j}=0, \quad i=1, \ldots, p+1 .
$$

The following result then generalizes Proposition 4.3 in Hallin, Ingenbleek and Puri (1985).

Proposition 2.3. $n^{1 / 2}\left(S^{(n)}-m^{(n)}\right)$ is asymptotically normal, with mean 0 and variance $V^{2}$ under $H_{0}^{(n)}$, with mean $\sum_{i=1}^{p}\left(a_{i}+b_{i}\right) C_{i}$ and variance $V^{2}$ under $K^{(n)}(\mathbf{a}, \mathbf{b} ; f)$, where

$$
\begin{aligned}
V^{2}=\int_{[0,1]^{2 p+1}}\{[ & {\left[\left(v_{1}, \ldots, v_{p+1}\right)\right]^{2} } \\
& \left.+2 \sum_{j=1}^{p} J\left(v_{1}, \ldots, v_{p+1}\right) J\left(v_{1+j}, \ldots, v_{p+1+j}\right)\right\} d v_{1} \cdots d v_{2 p+1}
\end{aligned}
$$

and

$$
C_{i}=\int_{[0,1]^{p+1}} J\left(v_{1}, \ldots, v_{p+1}\right) \sum_{j=0}^{p-i} \phi\left(F^{-1}\left(v_{1+j}\right)\right) F^{-1}\left(v_{1+j+i}\right) d v_{1} \cdots d v_{p+1} .
$$

For the proof, see Appendix 3.

Notice that this result implies that the asymptotic distribution of $S^{(n)}$ is exactly the same under $K^{(n)}(\mathbf{a}, \mathbf{b} ; f)$ as under the $p$ th-order truncation 
of $K^{(n)}(\mathbf{a}, \mathbf{b} ; f)$ obtained by putting $0=a_{p+1}=a_{p+2}=\cdots$ and $0=b_{p+1}=$ $b_{p+2}=\cdots$ in (2.1). As a consequence, a finite-order serial rank statistic generally cannot capture all the available information for testing $H_{0}^{(n)}$ against $K^{(n)}(\mathbf{a}, \mathbf{b} ; f)$.

Accordingly unless $\mathbf{a}$ and $\mathbf{b}$ are such that $a_{i}=0=b_{i}$ for $i$ larger than some $p<\infty$ [in which case the general linear alternative $K^{(n)}(\mathbf{a}, \mathbf{b} ; f)$ reduces to the $\operatorname{ARMA}\left(p_{1}, q_{1}\right)$ alternatives considered in Hallin, Ingenbleek and Puri (1985)], the asymptotically most powerful test statistic for $H_{0}^{(n)}$ against $K^{(n)}(\mathbf{a}, \mathbf{b} ; f)$ does not belong to the class of linear serial rank statistics anymore. Yet an asymptotically most powerful test based on ranks does exist, as asserted by Proposition 2.4, but its "order" increases with sample size $n$.

Define the rank autocorrelation coefficient associated with density $f$ (or $f$-rank autocorrelation) of order $i$ as

$$
r_{i ; f}^{(n)}=\left[(n-i)^{-1} \sum_{t=i+1}^{n} \phi\left(F^{-1}\left(\frac{R_{t}^{(n)}}{n+1}\right)\right) F^{-1}\left(\frac{R_{t-i}^{(n)}}{n+1}\right)-m^{(n)}\right] / s^{(n)}
$$

with

$$
m^{(n)}=(n(n-1))^{-1} \sum_{1 \leq i_{1} \neq i_{2} \leq n} \phi\left(F^{-1}\left(\frac{i_{1}}{n+1}\right)\right) F^{-1}\left(\frac{i_{2}}{n+1}\right)
$$

and

$$
\begin{aligned}
{\left[s^{(n)}\right]^{2}=} & \frac{1}{n(n-1)} \sum_{1 \leq i_{1} \neq i_{2} \leq n}\left[\phi\left(F^{-1}\left(\frac{i_{1}}{n+1}\right)\right) F^{-1}\left(\frac{i_{2}}{n+1}\right)\right]^{2} \\
& +2 \frac{(n-2 i)}{(n-i)} \frac{1}{n(n-1)(n-2)} \sum_{1 \leq i_{1} \neq i_{2} \neq i_{3} \leq n}\left[\phi\left(F^{-1}\left(\frac{i_{1}}{n+1}\right)\right)\right. \\
& \left.\quad \times \phi\left(F^{-1}\left(\frac{i_{2}}{n+1}\right)\right) F^{-1}\left(\frac{i_{2}}{n+1}\right) F^{-1}\left(\frac{i_{3}}{n+1}\right)\right] \\
& +\frac{n^{2}-n(2 i+3)+i^{2}+5 i}{n(n-1)(n-2)(n-3)(n-i)} \underset{1 \leq i_{1} \neq i_{2} \neq i_{3} \neq i_{4} \leq n}{n}\left[\phi\left(F^{-1}\left(\frac{i_{1}}{n+1}\right)\right)\right. \\
& \left.\quad \times \phi\left(F^{-1}\left(\frac{i_{2}}{n+1}\right)\right) F^{-1}\left(\frac{i_{3}}{n+1}\right) F^{-1}\left(\frac{i_{4}}{n+1}\right)\right] \\
& -(n-i)\left(m^{(n)}\right)^{2} .
\end{aligned}
$$

If $\mathbf{a}$ and $\mathbf{b}$ belong to $l^{2}$, we denote by $\langle\mathbf{a}, \mathbf{b}\rangle=\sum_{i=1}^{\infty} a_{i} b_{i}$ their scalar product; $\|\mathbf{a}\|=\langle a, a\rangle^{1 / 2}$ stands for the corresponding norm.

Proposition 2.4. Consider the test statistic

$$
S^{(n) *}=n^{-1 / 2} \sum_{i=1}^{n-1}(n-i)^{1 / 2}\left(a_{i}+b_{i}\right) r_{i ;}^{(n)} /\left[\sum_{i=1}^{n-1}\left(a_{i}+b_{i}\right)^{2}\right]^{1 / 2} .
$$


Then:

(i) $n^{1 / 2} S^{(n) *}$ is asymptotically normal, with mean 0 and variance 1 under $H_{0}^{(n)}$, and with mean $\langle(\mathbf{a}+\mathbf{b}),(\tilde{\mathbf{a}}+\tilde{\mathbf{b}})\rangle\left[\sigma^{2} I(f)\right]^{1 / 2} /\|\mathbf{a}+\mathbf{b}\|$ and variance 1 under $K^{(n)}(\tilde{\mathbf{a}}, \tilde{\mathbf{b}} ; f)\left(\right.$ with $\left.\tilde{\mathbf{a}}, \tilde{\mathbf{b}} \in l^{2}\right)$.

(ii) $S^{(n) *}$ provides an asymptotically most powerful test for $H_{0}^{(n)}$ against the general linear alternative $K^{(n)}(\mathbf{a}, \mathbf{b} ; f)$ (among all tests of given level $\left.\alpha\right)$ :

$$
\begin{aligned}
\varphi_{\alpha}^{(n) *}(\mathbf{a}, \mathbf{b} ; f) & =1 \\
\text { if } \sum_{i=1}^{n-1}(n-i)^{1 / 2}\left(a_{i}+b_{i}\right) r_{i ; f}^{(n)} & >\left[\sum_{i=1}^{n-1}\left(a_{i}+b_{i}\right)^{2}\right]^{1 / 2} k_{1-\alpha},
\end{aligned}
$$

where $k_{1-\alpha}$ denotes the $(1-\alpha)$-quantile of the standard normal distribution.

For the proof, see Appendix 3.

REMARK 2.1. Unless there exists some $p<\infty$ such that $a_{i}+b_{i}=0, i>p$ [in which case (2.9) reduces to (1.3)], $S^{(n)^{*}}$ does not belong to the class of linear serial rank statistics anymore. Its asymptotic distribution cannot be obtained by the same techniques [viz. score-generating functions) as in Hallin, Ingenbleek and Puri (1985). Call $S^{(n)^{*}}$ a generalized linear serial rank statistic.

REMARK 2.2. The asymptotic distribution of $n^{1 / 2} S^{(n)^{*}}$-hence the asymptotic optimality of the corresponding test (2.9)-depends on $\mathbf{a}$ and $\mathbf{b}$ through the sum $\mathbf{a}+\mathbf{b}$ only: (2.9) is therefore asymptotically most powerful against the more general alternative $U\left\{K^{(n)}(\alpha, \beta ; f) \mid \alpha+\beta=\mathbf{a}+\mathbf{b}\right\}$.

REMARK 2.3. Just as in the case of finite-order ARMA alternatives, there exist general linear alternatives $K^{(n)}(\tilde{\mathbf{a}}, \tilde{\mathbf{b}} ; f)$ against which the asymptotic power of (2.9) is $\alpha$ (viz. those for which $(\tilde{\mathbf{a}}+\tilde{\mathbf{b}}) \perp(\mathbf{a}+\mathbf{b})$, where $\perp$ denotes the orthogonality relation in $l^{2}$ ]. This motivates the consideration of generalized quadratic rank statistics (see Section 4.1).

\section{Testing an ARMA model against another ARMA model: Asymptotic sufficiency.}

3.1. The general linear alternative for residuals. Consider now the null hypothesis $H^{(n)}(\mathbf{A}, \mathbf{B} ; \cdot)$ under which the observed series is generated by the $\operatorname{ARMA}\left(p_{1}, q_{1}\right)$ model (1.7), where

(a) $A(L)$ and $B(L)$ are of orders $p_{1}$ and $q_{1}$, respectively (i.e., $A_{p_{1}} \neq 0 \neq B_{q_{1}}$ ),

(b) the polynomials $A(z)$ and $B(z), z \in \mathbb{C}$, have no common roots,

(c) the polynomials $A(z)$ and $B(z), z \in \mathbb{C}$, have all their roots outside the unit circle,

(d) the unspecified density $f(\cdot)$ of $\left\{\varepsilon_{t}\right\}$ satisfies the assumptions of Section 2.1 .

Denoting by $Z_{t}$ the filtered observations $(A(L) / B(L)) X_{t}$ [i.e., the observed residuals with respect to (1.7)], we have seen that, under the null hypothesis, 
$\left\{Z_{t}\right\}$ is a white noise process. Let us show that, under the alternative $K^{(n)}(\mathbf{A}, \mathbf{B} ; \gamma, \delta ; f)$ [see (1.8) and (1.9)], $\left\{Z_{t}\right\}$ is generated by a general linear model of the form (2.1) we investigated in Section 2. We shall assume that $\alpha^{(n)}(L)$ and $\beta^{(n)}(L)$

(e) are such that the polynomials $\alpha^{(n)}(z)$ and $\beta^{(n)}(z), z \in \mathbb{C}$, have no root which is common for any value of $n \in \mathbb{N}$.

For $n$ sufficiently large, $\alpha^{(n)}(z)$ and $\beta^{(n)}(z)$ have all their roots outside the unit circle and (1.8) uniquely determines a sequence of stationary ARMA processes; of course, we still assume that the density function $f(\cdot)$ of $\left\{\varepsilon_{t}\right\}$ satisfies the assumptions of Section 2.1. Suppose $p_{1}>0$ and $q_{1}>0$, and denote by $G_{u}$ and $H_{u}, u \in \mathbb{Z}$, the Green's functions of the operators $A(L)$ and $B(L)$, respectively (for definition, see Appendix 1). Under the preceding assumptions (a), (b) and (c), we have $[A(L)]^{-1}=1+\sum_{u=1}^{\infty} G_{u} L^{u}$ and $[B(L)]^{-1}=1+\sum_{u=1}^{\infty} H_{u} L^{u}$, with $\sum_{u=1}^{\infty}\left|G_{u}\right|<\infty$ and $\sum_{u=1}^{\infty}\left|H_{u}\right|<\infty$; (1.10) then can be written as

$$
\begin{aligned}
{[1-} & \left.n^{-1 / 2} \sum_{u=1}^{\infty} \sum_{i=1}^{\min \left(p_{2}, u\right)} \gamma_{i} G_{u-i} L^{u}\right] Z_{t} \\
& =\left[1+n^{-1 / 2} \sum_{u=1}^{\infty} \sum_{i=1}^{\min \left(q_{2}, u\right)} \delta_{i} H_{u-i} L^{u}\right] \varepsilon_{t} .
\end{aligned}
$$

Let

$$
a_{i}=\sum_{j=1}^{\min \left(p_{2}, i+p_{1}-1\right)} \gamma_{j} G_{i-j}
$$

and

$$
b_{i}=\sum_{j=1}^{\min \left(q_{2}, i+q_{1}-1\right)} \delta_{j} H_{i-j}, \quad i=1,2, \ldots
$$

Because $G_{-1}=G_{-2}=\cdots=G_{-p_{1}+1}=H_{-1}=H_{-2}=\cdots=H_{-q_{1}+1}=0,(3.1)$ is of the form (2.1). Let us show, moreover, that $\sum_{i=1}^{\infty}\left|a_{i}\right|<\infty$ and $\sum_{i=1}^{\infty}\left|b_{i}\right|<\infty$. For $i \leq p_{2}-p_{1}+1$, the $a_{i}$ 's are a linear combination of $G_{i-1}, G_{i-2}, \ldots, G_{i-p_{2}}$, which are solutions of the homogeneous equation $A(L) G_{t}=0, t \in \mathbb{Z}$; because of assumption (c), such solutions are decaying to zero, as $i \rightarrow \infty$, at an exponential rate, which implies that $\sum_{i=1}^{\infty}\left|a_{i}\right|$ converges. The same holds for the $b_{i}$ 's and operator $B(L)$. Equation (3.1) thus constitutes a particular case of a general linear alternative, and testing $H^{(n)}(\mathbf{A}, \mathbf{B} ; \cdot)$ against $K^{(n)}(\mathbf{A}, \mathbf{B} ; \boldsymbol{\gamma}, \delta ; f)$ reduces to the problem of testing $H_{0}^{(n)}$ against $K^{(n)}(\mathbf{a}, \mathbf{b} ; f)$, with $\mathbf{a}$ and $\mathbf{b}$ given in (3.2).

If $p_{1}=0$ and/or $q_{1}=0$, put $a_{i}=\gamma_{i}, i=1, \ldots, p_{2}$, and/or $b_{i}=\delta_{i}, i=$ $1, \ldots, q_{2}$.

3.2. Asymptotically most powerful test and asymptotic sufficiency of f-rank autocorrelations. An asymptotically most powerful test for $H^{(n)}(\mathbf{A}, \mathbf{B} ; \cdot)$ 
[ARMA model (1.7)] against $K^{(n)}(\mathbf{A}, \mathbf{B} ; \gamma, \delta ; f)$ [alternative model (1.8)] can now straightforwardly be obtained from Proposition 2.4 .

Proposition 3.1. An asymptotically most powerful test for $H^{(n)}(\mathbf{A}, \mathbf{B} ; \cdot)$ against $K^{(n)}(\mathbf{A}, \mathbf{B} ; \gamma, \delta ; f)$ is given by $(2.9)$, where the rank autocorrelations $r_{i ; f}^{(n)}$ given by (2.5) are computed from the ranks of the filtered series $\mathbf{Z}^{(n)}$, and the $a_{i}$ 's and $b_{i}$ 's are given in (3.2). Under the same conditions, the asymptotic distribution of the generalized linear serial rank statistic (2.8) remains the same as in Proposition 2.4(i).

Unless otherwise specified, all the ranks and rank statistics we refer to in the sequel are computed from the filtered series $\mathbf{Z}^{(n)}$.

Consider the general problem of testing $H^{(n)}$ against $K^{(n)}$, where $H^{(n)}=\left\{l_{0}^{(n)}\right\}$ and $K^{(n)}=\left\{l_{1}^{(n)}\right\}$ are families of densities for some observed series $\mathbf{X}^{(n)}$ defined over a sample space $\left(\mathscr{X}^{(n)}, \mathscr{A}^{(n)}\right)$. Recall that the envelope power function $\beta\left(\alpha, H^{(n)}, K^{(n)}\right)$ for this problem is defined as

$$
\beta\left(\alpha, H^{(n)}, K^{(n)}\right)=\sup _{\psi^{(n)}} \inf _{l_{1}^{(n)} \in K^{(n)}} E_{l_{1}^{(n)}}\left[\psi^{(n)}\right],
$$

where the supremum is taken over all the tests $\psi^{(n)}$ satisfying, for fixed $\alpha \in(0,1)$,

$$
E_{l_{0}^{(n)}}\left[\psi^{(n)}\right] \leq \alpha, \quad l_{0}^{(n)} \in H^{(n)} .
$$

Let $T^{(n)}$ be some statistic from $\left(\mathscr{X}^{(n)}, \mathscr{A}^{(n)}\right)$ to some range space $\left(\mathscr{T}^{(n)}, \mathscr{B}^{(n)}\right)$. Define the envelope power function $\beta_{T}\left(\alpha, H^{(n)}, K^{(n)}\right)$ associated with $T^{(n)}$ by taking the supremum in (3.3) over the set of all $T^{(n)}$-measurable tests $\psi^{(n)}$ satisfying (3.4). If we denote by $H_{T}^{(n)}$ and $K_{T}^{(n)}$ the families of densities [on $\left(\mathscr{T}^{(n)}, \mathscr{B}^{(n)}\right)$ ] induced from $H^{(n)}$ and $K^{(n)}$ by $T^{(n)}$, then clearly $\beta_{T}\left(\alpha, H^{(n)}, K^{(n)}\right)=\beta\left(\alpha, H_{T}^{(n)}, K_{T}^{(n)}\right)$. Obviously, we also have

$$
\beta\left(\alpha, H^{(n)}, K^{(n)}\right) \geq \beta_{T}\left(\alpha, H^{(n)}, K^{(n)}\right) ;
$$

if, however, an equality in (3.5) is achieved for every $\alpha \in(0,1)$ [this happens, e.g., if $T^{(n)}$ is sufficient for the structure $\left.\left(\mathscr{X}^{(n)}, \mathscr{A}^{(n)}, H^{(n)} \cup K^{(n)}\right)\right]$, then $T^{(n)}$ can be considered, in some sense, as sufficient for the problem of testing $H^{(n)}$ against $K^{(n)}$. Accordingly, [Hájek and Sidák (1967), Chapter 7], we say that $T^{(n)}$ is asymptotically sufficient for the problem of testing $H^{(n)}$ against $K^{(n)}$ if

$$
\lim _{n \rightarrow \infty}\left[\beta\left(\alpha, H^{(n)}, K^{(n)}\right)-\beta_{T}\left(\alpha, H^{(n)}, K^{(n)}\right)\right]=0, \quad \alpha \in(0,1) .
$$

An important consequence is that if $T^{(n)}$ is asymptotically sufficient for $H^{(n)}$ against $K^{(n)}$, then any asymptotically maximin most powerful test $\psi^{(n)^{*}}$ for $H_{T}^{(n)}$ against $K_{T}^{(n)}$ is also asymptotically maximin most powerful for $H^{(n)}$ against $K^{(n)}$.

Let us consider now the problem of testing $H^{(n)}(\mathbf{A}, \mathbf{B} ; \cdot)$ against unspecified ARMA alternatives. Denote by

$$
K^{(n)}\left(\mathbf{A}, \mathbf{B} ; \mathbb{R}^{p_{2}}, \mathbb{R}^{q_{2}} ; f\right)=\bigcup\left\{K^{(n)}(\mathbf{A}, \mathbf{B} ; \gamma, \delta ; f) \mid \gamma \in \mathbb{R}^{p_{2}}, \boldsymbol{\delta} \in \mathbb{R}^{q_{2}}\right\}
$$


the alternative under which the observed series $\mathbf{X}^{(n)}$ is generated by some ARMA model [(1.8) and (1.9)] where $\gamma$ and $\delta$ take on unspecified values, although being of specified dimensions $p_{2}$ and $q_{2}$. Let also $d \in \mathbb{R}^{+}$, and denote by $\mathscr{D}(d)$ the set of values of $(\gamma, \boldsymbol{\delta}) \in \mathbb{R}^{p_{2}} \times \mathbb{R}^{q_{2}}$ such that $\|\mathbf{a}+\mathbf{b}\| \geq d$, with a and $b$ given in (3.2). In order to obtain asymptotically maximin most powerful tests, we shall need to consider subhypotheses of the form

$$
K^{(n)}\left(\mathbf{A}, \mathbf{B} ; \mathbb{R}^{p_{2}}, \mathbb{R}^{q_{2}} ; f \mid d\right)=\bigcup\left\{K^{(n)}(\mathbf{A}, \mathbf{B} ; \gamma, \delta ; f) \mid(\gamma, \delta) \in \mathscr{D}(d)\right\} .
$$

We then have the following sufficiency result.

Proposition 3.2. Let $\left\{\Psi_{t}^{(1)}, \ldots, \Psi_{t}^{\left(p_{1}+q_{1}\right)}\right)$ denote an arbitrary fundamental system of solutions of the homogeneous equation $A(L) B(L) \Psi_{t}=0, t \in \mathbb{Z}$. Writing $\pi$ for $\max \left(p_{2}-p_{1}, q_{2}-q_{1}\right)$, consider the $\max \left(p_{1}+q_{2}, p_{2}+q_{1}\right)$-tuple of rank statistics [assume $n \geq \max \left(p_{2}-p_{1}+3, q_{2}-q_{1}+3, p_{1}+q_{1}+2\right)$ ]

$$
\mathbf{T}_{\Psi ; f}^{(n)}=\left(r_{1 ; f}^{(n)}, \ldots, r_{\pi ; f}^{(n)}, \sum_{i=\pi+1}^{n-1} \Psi_{i}^{(1)} r_{i ; f}^{(n)}, \ldots, \sum_{i=\pi+1}^{n-1} \Psi_{i}^{\left(p_{1}+q_{1}\right)} r_{i ; f}^{(n)}\right)
$$

Then, for any $d \in \mathbb{R}^{+}, \mathbf{T}_{\Psi ; f}^{(n)}$ is asymptotically sufficient for the problem of testing $H^{(n)}(\mathbf{A}, \mathrm{B} ; \cdot)$ against $(3.8)$.

The proof (see Appendix 3) consists in establishing that the sufficient conditions for asymptotic sufficiency of Hájek and Šidák's (1967) Theorem 7.1.1 are satisfied.

3.3. Asymptotic distribution of $\mathbf{T}_{\Psi ; f}^{(n)}$. We conclude this section by establishing the asymptotic multinormality of any asymptotically sufficient vector $\mathbf{T}_{\Psi ; f}^{(n)}$. The proof of the following proposition essentially follows along the same lines as in Proposition 2.4 and is only briefly sketched in Appendix 3.

Proposition 3.3. $n^{1 / 2} \mathbf{T}_{\Psi ; f}^{(n)}$ is asymptotically normal, with mean $\mathbf{0}$ under $H^{(n)}(\mathbf{A}, \mathbf{B} ; \cdot)$, mean $\left(\left(a_{1}+b_{1}\right), \ldots, \quad\left(a_{\pi}+b_{\pi}\right), \quad \sum_{i=\pi+1}^{\infty} \Psi_{i}^{(1)}\left(a_{i}+b_{i}\right)\right.$, $\left.\ldots, \sum_{i=\pi+1}^{\infty} \Psi_{i}^{\left(p_{1}+q_{1}\right)}\left(a_{i}+b_{i}\right)\right)^{\prime}\left[\sigma^{2} I(f)\right]^{1 / 2}$ under $K^{(n)}(\mathbf{A}, \mathbf{B} ; \gamma, \delta ; f)$ and full-rank covariance matrix

$$
\underline{\mathbf{W}}_{\Psi}^{2}=\left(\begin{array}{cc}
\mathbf{I}_{\pi \times \pi} & \mathbf{0} \\
\mathbf{0} & \mathbf{W}_{\Psi}^{2}
\end{array}\right)
$$

under both where $\mathbf{W}_{\Psi}^{2}=\left(W_{\Psi ; k l}\right)$ and $W_{\Psi ; k l}=\sum_{i=\pi+1}^{\infty} \Psi_{i}^{(k)} \Psi_{i}^{(l)}<\infty, \quad k, l=$ $1, \ldots, p_{1}+q_{1}$.

4. Testing an ARMA model against another ARMA model: Asymptotically maximin most powerful tests.

4.1. Generalized quadratic serial rankstatistics. Quadratic serial rank statistics, providing rank-based versions of Box and Pierce's (1970) portmanteau statistic were introduced in Hallin, Ingenbleek and Puri (1987). Since 
however the asymptotically most powerful test for $H^{(n)}(\mathbf{A}, \mathbf{B} ; \cdot)$ against $K^{(n)}(\mathbf{A}, \mathbf{B} ; \boldsymbol{\gamma}, \boldsymbol{\delta} ; f)$ cannot, in general, be found within the class of linear serial rank tests, quadratic serial rank statistics cannot be expected to provide optimal tests against unspecified values of $\gamma$ and $\delta$ (unless, e.g., $p_{1}=0=q_{1}$ ), and a more general type of quadratic rank statistics has to be considered: generalized quadratic serial rank statistics.

Let $\mathbf{S}^{(n)}-\mathbf{m}^{(n)}=\left(S_{1}^{(n)}-m_{1}^{(n)}, \ldots, S_{l}^{(n)}-m_{l}^{(n)}\right)^{\prime}$ be a column vector of generalized linear serial rank statistics centered about their means [under $\left.H^{(n)}(\mathbf{A}, \mathbf{B} ; \cdot)\right]$. Assuming that $n^{1 / 2}\left(\mathbf{S}^{(n)}-\mathbf{m}^{(n)}\right)$ has an asymptotic covariance matrix $\mathbf{V}^{2}$ of full rank, we define a generalized quadratic serial rank statistic as a statistic of the form $Q^{(n)}=n\left(\mathbf{S}^{(n)}-\mathbf{m}^{(n)}\right)^{\prime} \mathbf{V}^{-2}\left(\mathbf{S}^{(n)}-\mathbf{m}^{(n)}\right)$.

Since we have shown that asymptotically sufficient statistics $\mathbf{T}_{\Psi ; f}^{(n)}$ exist for testing $H^{(n)}(\mathbf{A}, \mathbf{B} ; \cdot)$ against $K^{(n)}(\mathbf{A}, \mathbf{B} ; \gamma, \delta ; f)$, it is intuitively quite natural to consider generalized quadratic serial rank statistics of the form

$$
Q_{A, B ; f}^{(n) *}=n \mathbf{T}_{\Psi ; f}^{(n),} \mathbf{W}_{\Psi}^{-2} \mathbf{T}_{\Psi ; f}^{(n)} .
$$

Actually, we shall establish in Section 4.2 that $Q_{A, B ;}^{(n) *}$ provides an asymptotically maximin most powerful test for our problem. We first justify the notation by establishing the important property that $Q_{A, B ; f}^{(n) *}$, unlike $\mathbf{T}_{\Psi ; f}^{(n)}$, does not depend on the particular fundamental system $\left\{\Psi_{t}^{(i)}\right\}$ adopted.

Proposition 4.1. The quadratic statistic $Q_{A, B ; f}^{(n)^{*}}$ does not depend on the fundamental system $\left\{\Psi_{t}^{(i)}\right\}$.

For the proof, see Appendix 3.

Let us illustrate Proposition 4.1 by means of two examples.

ExAmple 4.1. Denote by $\lambda_{(1)} \cdots \lambda_{\left(p_{1}+q_{1}\right)}$ the roots of $A\left(z^{-1}\right) B\left(z^{-1}\right)=0$, $z \in \mathbb{C}$, and assume that they all are distinct (hence of multiplicity 1 -also recall that they all lie inside the unit circle). Then a fundamental system of solutions is provided by $\left\{\Psi_{t}^{(j)}=\lambda_{j}^{t-(n+1)}, t \in \mathbb{Z} ; j=1, \ldots, p_{1}+q_{1}\right\} . Q_{A, B}^{(n)^{*}}$ is thus a quadratic form involving the rank autocorrelations of orders 1 through $\pi$ and geometrically weighted sums of rank autocorrelations of orders $\pi+1$ through $n-1 . \mathbf{W}_{\Psi}^{2}$ is then given by $\left(W_{\Psi ; k l}\right)=\left(\left(1-\lambda_{k} \lambda_{l}\right)^{-1}\right)$.

EXAMPLE 4.2. Denote by $\mathscr{G}_{u}=\mathscr{G}(t, t-u)$ the Green's functions associated with the difference operator $A(L) B(L)$. Then (see Appendix 1) another fundamental system of solutions is $\left\{\Psi_{t}^{(j)}=\mathscr{G}_{t-\pi-j}, \quad t \in \mathbb{Z} ; j=1, \ldots, p_{1}+q_{1}\right\}$. Recalling that $\mathscr{G}_{-1}=\mathscr{G}_{-2}=\cdots=\mathscr{G}_{-p_{1}-q_{1}+1}=0$, we obtain $W_{\Psi ; k l}=$ $\sum_{i=0}^{\infty} \mathscr{G}_{-k-1+i} \mathscr{G}_{-l-1+i}$, hence

$$
\mathbf{W}_{\Psi}^{2}=\left(\begin{array}{ccc}
\gamma^{(0)} \gamma^{(1)} & \cdots & \gamma^{\left(p_{1}+q_{1}-1\right)} \\
\gamma^{(1)} & \ddots & \vdots \\
\vdots & \ddots & \gamma^{(1)} \\
\gamma^{\left(p_{1}+q_{1}-1\right)} & \cdots & \gamma^{(1)} \gamma^{(0)}
\end{array}\right),
$$


where we denote by $\gamma^{(0)}, \ldots, \gamma^{\left(p_{1}+q_{1}-1\right)}$ the first $p_{1}+q_{1}-1$ autocovariances of the $\operatorname{AR}\left(p_{1}+q_{1}\right)$ process $\left\{\xi_{t} ; t \in \mathbb{Z}\right\}$ characterized by the $\operatorname{AR}\left(p_{1}+q_{1}\right)$ model

$$
A(L) B(L) \xi_{t}=\eta_{t}, \quad t \in \mathbb{Z}
$$

$\left[\left(\eta_{t} ; t \in \mathbb{Z}\right)\right.$ a second-order standardized white noise $]$. This matrix $\mathbf{W}_{\Psi}^{2}$, which has also the nature of a Casorati matrix (cf. Appendix 1), is the one that appears in the usual Yule-Walker equations associated with (4.2).

We now give the asymptotic distribution of $Q_{A, B ; f}^{(n)^{*}}$.

Proposition 4.2. Under $H^{(n)}(\mathbf{A}, \mathbf{B} ; \cdot), Q_{A, B ; f}^{(n)^{*}}$ is asymptotically $\chi^{2}$ with $\max \left(p_{1}+q_{2}, p_{2}+q_{1}\right)$ degrees of freedom. Under $K^{(n)}(\mathbf{A}, \mathbf{B} ; \gamma, \delta ; f)$, it is asymptotically noncentral $\chi^{2}$, with the same degrees of freedom and with noncentrality parameter

$$
\lambda_{f}^{*}(\gamma, \boldsymbol{\delta})=\frac{1}{2}\|\mathbf{a}+\mathbf{b}\|^{2} \mathbf{\sigma}^{2} I(f),
$$

where $\mathbf{a}$ and $\mathbf{b}$ are given by (3.2).

For the proof, see Appendix 3.

4.2. Asymptotically maximin most powerful tests for testing an ARMA model against unspecified ARMA alternatives. Let us define an asymptotically maximin most powerful test for $H^{(n)}(\mathbf{A}, \mathbf{B} ; \cdot)$ against an unspecified $\operatorname{ARMA}\left(p_{2}, q_{2}\right)$ alternative as a test which is asymptotically maximin most powerful (among all tests of the same level) against any alternative of the form $K^{(n)}\left(\mathbf{A}, \mathbf{B} ; \mathbb{R}^{p_{2}}\right.$, $\left.\mathbb{R}^{q_{2}} ; f \mid d\right)$ [cf. (3.8)], $d \in \mathbb{R}^{+}, f$ specified up to a scale parameter. We then have the following result.

Proposition 4.3. An asymptotically maximin most powerful test for $H^{(n)}(\mathbf{A}, \mathbf{B} ; \cdot)$ against an unspecified $A R M A\left(p_{2}, q_{2}\right)$ alternative is provided by

$$
\psi_{\alpha}^{(n)^{*}}\left(\mathbf{A}, \mathbf{B} ; p_{2}, q_{2} ; f\right)=1 \quad \text { if } Q_{A, B ; f}^{(n)^{*}}>\chi_{\max \left(p_{1}+q_{2}, p_{2}+q_{1}\right) ; 1-\alpha}^{2} .
$$

Accordingly, the envelope power function

$$
\beta\left(\alpha, H^{(n)}(\mathbf{A}, \mathbf{B} ; \cdot), K^{(n)}\left(\mathbf{A}, \mathbf{B} ; \mathbb{R}^{p_{2}}, \mathbb{R}^{q_{2}} ; f \mid d\right)\right)
$$

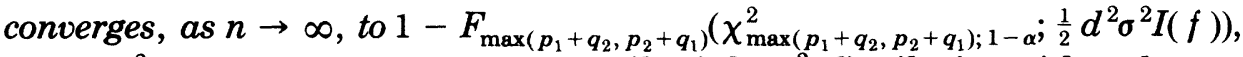
where $\chi_{m ; 1-\alpha}^{2}$ denotes the $(1-\alpha)$-quantile of the $\chi^{2}$ distribution with $m$ degrees of freedom and $F_{m}(\cdot ; \lambda)$ the distribution function of the noncentral $\chi^{2}$ with $m$ degrees of freedom and noncentrality parameter $\lambda$.

CoRollary 4.4. The rank portmanteau statistic $n \sum_{i=1}^{p}\left(r_{i ; f}^{(n)}\right)^{2}$ (cf. Hallin, Ingenbleek and Puri, 1987) is asymptotically maximin most powerful against unspecified $\operatorname{ARMA}\left(p_{2}, q_{2}\right)$ alternatives if and only if $p_{1}=q_{1}=0$ and $p=$ $\max \left(p_{2}, q_{2}\right)$.

For the proof, see Appendix 3. 
REMARK 4.1. Denoting by $Q_{f}^{(n)}=n \sum_{i=1}^{\pi}\left(r_{i ; f}^{(n)}\right)^{2}$ the rank portmanteau statistic of order $\pi=\max \left(p_{2}-p_{1}, q_{2}-q_{1}\right)$, maximin-optimal quadratic statistics $Q_{A, B ; f}^{(n)^{*}}$ can be decomposed into $Q_{A, B ; f}^{(n) *}=Q_{f}^{(n)}+Q_{A, B ; f}^{(n)}$. Because $Q_{f}^{(n)}$ only depends on $r_{1 ; f}^{(n)}, \ldots, r_{\pi ; f}^{(n)}$ whereas $Q_{A, B ; f}^{(n)}$ only depends on $r_{\pi+1 ; f}^{(n)}, r_{\pi+2 ; f}^{(n)}, \ldots$, $Q_{f}^{(n)}$ and $Q_{A, B ; f}^{(n)}$ are asymptotically independent $\chi^{2}$-distributed statistics [under $\left.H^{(n)}(\mathrm{A}, \mathrm{B} ; \cdot)\right] . Q_{\}}^{(n)}$ contributes for $\max \left(p_{2}-p_{1}, q_{2}-q_{1}\right)$ degrees of freedom, the maximal number of possible higher-order terms (autoregressive terms of orders larger than $p_{1}$ and/or moving average terms of orders larger than $q_{1}$ ). $Q_{A, B ; f}^{(n)}$ has the form of a weighted rank portmanteau statistic and contributes for $\left(p_{1}+q_{1}\right)$ degrees of freedom, one for each tested coefficient $A_{1}, \ldots, A_{p_{1}}$, $B_{1}, \ldots, B_{q_{1}}$. The consideration of this decomposition in case $H^{(n)}(\mathbf{A}, \mathbf{B} ; \cdot)$ has been rejected, may provide a useful insight into the reasons why rejection occurred, and thus suggest alternative model specifications.

REMARK 4.2. The noncentrality parameter $\lambda_{f}^{*}(\gamma, \delta)$ of the asymptotic distribution of $Q_{A, B ; f}^{(n) *}$ under alternatives of the form $K^{(n)}(A, B ; \gamma, \delta ; f)$ also can be decomposed into $\frac{1}{2} \sum_{i=1}^{\pi}\left(a_{i}+b_{i}\right)^{2} \sigma^{2} I(f)$ (contribution of the "unweighted part" $Q_{f}^{(n)}$ ) and $\frac{1}{2} \sum_{i=\pi+1}^{\infty}\left(a_{i}+b_{i}\right)^{2} \sigma^{2} I(f)$ (contribution of the "weighted part" $\left.Q_{A, B ; f}^{(n)}\right)$. A study of the relation between these two quantities and the coefficients of the tested model (1.7) provides an interesting insight into the importance of the respective contributions of the "weighted" and "unweighted" parts to the asymptotic power of (4.4). As a general rule, the contribution of the "unweighted part" does not depend on $A(L)$ and $B(L)$, whereas the contribution of the "weighted part" is an increasing function of the "closeness to the unit circle" of the roots of $A(z) B(z)=0, z \in \mathbb{C}$. To show this, let us consider a simple example.

ExAmple 4.3. Consider the problem of testing the AR(1) model $X_{t}-$ $\rho X_{t-1}=\varepsilon_{t}, t \in \mathbb{Z}$, where $|\rho|<1$, against $\operatorname{ARMA}(2,1)$ alternatives. Here $\pi=1$ and

$$
\begin{aligned}
a_{i} & = \begin{cases}\gamma_{1}, & i=1, \\
\gamma_{1} \rho^{i-1}+\gamma_{2} \rho^{i-2}, & i \geq 2,\end{cases} \\
b_{i} & = \begin{cases}\delta_{1}, & i=1, \\
0, & i \geq 2,\end{cases}
\end{aligned}
$$

if an alternative of the form $K^{(n)}\left(\mathbf{A}, \mathbf{B} ; \boldsymbol{\gamma}=\left(\gamma_{1}, \gamma_{2}\right), \delta=\left(\delta_{1}, 0\right) ; f\right)$ is considered. The noncentrality parameter (4.3) accordingly decomposes into $\left(\gamma_{1}+\delta_{1}\right)^{2}$ (contribution of the "unweighted" $Q_{f}^{(n)}$ ), which does not depend on $\rho$, and $\sum_{i=2}^{\infty}\left(\gamma_{1} \rho^{i-1}+\gamma_{2} \rho^{i-2}\right)^{2}=\left(\gamma_{1} \rho+\gamma_{2}\right)^{2}\left(1 /\left(1-\rho^{2}\right)\right)$ (contribution of the "weighted" $\left.Q_{A, B ; f}^{(n)}\right)$. Clearly, this latter contribution is approximately $\gamma_{2}^{2}$ if $|\rho| \simeq 0$-and the optimal test (4.4) is then approximately equivalent to the test based on an "unweighted" rank portmanteau statistic of order 2 ; if $|\rho| \simeq 1$, then the contribution of $Q_{A, B ; f}^{(n)}$ can be arbitrarily large.

The absence of the weighted part $Q_{A, B ; f}^{(n)}$ in the classical portmanteau statistic is possibly responsible for the somewhat disappointing performance of the Box-Pierce test (see also Remark 4.3). 
REMARK 4.3. Corollary 4.4 characterizes the only case [viz. $A(L)=I=$ $B(L)]$ where an unweighted rank portmanteau statistic $Q_{f}^{(n)}$ is asymptotically maximin most powerful against the whole unspecified $\operatorname{ARMA}\left(p_{2}, q_{2}\right)$ alternative. $Q f^{(n)}$ can be shown however to provide maximin most powerful tests for more general model operators $A(L)$ and $B(L)$ if this alternative is restricted in an adequate manner. It can be shown, for example, that the unweighted rank portmanteau statistic of order $\pi$ is asymptotically maximin most powerful for testing $H^{(n)}(\mathbf{A}, \mathbf{B} ; \cdot)$ against the set of all $\operatorname{ARMA}\left(p_{2}, q_{2}\right)$ models of the form $A(L) a^{(n)}(L) X_{t}=B(L) b^{(n)}(L) \varepsilon_{t}$ considered in $(1.11)$, where $a^{(n)}(L)=1-$ $n^{-1 / 2}\left(a_{1} L+\cdots+a_{\pi} L^{\pi}\right)$ and $b^{(n)}(L)=1+n^{-1 / 2}\left(b_{1} L+\cdots+b_{\pi} L^{\pi}\right)$ are unspecified, i.e., against alternative models which differ from the tested one only because of the existence of $\pi$ additional roots to the characteristic equations.

This latter type of alternative generalizes the ones considered for purely autoregressive processes with Gaussian generating white noise by Hosking (1978), who shows that in this particular case the parametric Box-Pierce portmanteau test asymptotically coincides with the likelihood ratio test.

REMARK 4.4. $Q_{A, B ; f}^{(n) *}$ depends on $p_{2}$ and $q_{2}$ only through $\Delta=\max \left(p_{2}-\right.$ $\left.p_{1}, q_{2}-q_{1}\right) ;(4.4)$ is thus actually asymptotically maximin most powerful against the whole unspecified $\operatorname{ARMA}\left(p_{1}+\Delta, q_{1}+\Delta\right)$ alternative.

REMARK 4.5. $\quad Q_{A, B ; f}^{(n)^{*}}$ depends on the operators $A(L)$ and $B(L)$ only through the symbolic product $A(L) B(L)$, and thus takes the same form whether the tested model is $A(L) X_{t}=B(L) \varepsilon_{t}$ or $B(L) X_{t}=A(L) \varepsilon_{t}$ or $A(L) B(L) X_{t}=\varepsilon_{t}$, etc.; but the filtered series $\mathbf{Z}^{(n)}$ from which $Q_{A, B ; f}^{(n) *}$ has to be computed is not the same.

REMARK 4.6. The coefficients appearing in the "weighted part" $Q_{A, B ; f}^{(n)}$ of $Q_{A, B ; f}^{(n) *}$ (see Remark 4.1) are computed here from the asymptotic covariance matrix $\mathbf{W}^{2}$ of $\mathbf{T}_{\Psi ; f}^{(n)}$. For small sample sizes, an exact orthogonalizing matrix could possibly be introduced.

EXAMPLE 4.4. Consider the problem of testing the null hypothesis that an observed series $\mathbf{X}^{(n)}$ was generated by the $\operatorname{ARMA}(1,1)$ model $X_{t}-\frac{4}{5} X_{t-1}=$ $\varepsilon_{t}+\frac{1}{2} \varepsilon_{t-1}, t \in \mathbb{Z}$, against unspecified $\operatorname{ARMA}(2,2)$ alternatives [or, equivalently, against $\operatorname{ARMA}(2,1)$ or $\operatorname{ARMA}(1,2)$ alternatives; cf. Remark 4.4]. Let $Z_{-1}^{(n)}=$ $Z_{0}^{(n)}=0$ and $Z_{t}^{(n)}=X_{t}^{(n)}-\frac{4}{5} X_{t-1}^{(n)}-\frac{1}{2} Z_{t-1}^{(n)}, t=1, \ldots, n$; denote by $r_{i ;}^{(n)}$ the corresponding $f$-rank autocorrelations. A fundamental system of solutions of $\left(1-\frac{4}{5} L\right)\left(1+\frac{1}{2} L\right) \Psi_{t}=0, \quad t \in \mathbb{Z}$, is $\left\{\Psi_{t}^{(1)}=\left(\frac{4}{5}\right)^{t-2} ; \Psi_{t}^{(2)}=\left(-\frac{1}{2}\right)^{t-2}\right\}$ and $\pi=$ $\max \left(p_{2}-p_{1}, q_{2}-q_{1}\right)=1$. The covariance matrix $\underline{\mathbf{W}}^{2}$ (see Example 4.1) here is of the form

$$
\underline{\mathbf{W}}^{2}=\left(\begin{array}{cc}
{\left[1-\left(\frac{4}{5}\right)^{2}\right]^{-1}} & {\left[1+\frac{2}{5}\right]^{-1}} \\
{\left[1+\frac{2}{5}\right]^{-1}} & {\left[1-\left(\frac{1}{2}\right)^{2}\right]^{-1}}
\end{array}\right)=\left(\begin{array}{cc}
\frac{25}{9} & \frac{5}{7} \\
\frac{5}{7} & \frac{4}{3}
\end{array}\right)
$$


The statistic $Q_{A, B ; f}^{(n)^{*}}$ is thus

$$
\begin{aligned}
Q_{A, B ; f}^{(n)^{*}}= & n\left(r_{1 ; f}^{(n)}\right)^{2}+n\left(\sum_{i=2}^{n-1}\left(\frac{4}{5}\right)^{i-2} r_{i ; f}^{(n)}, \sum_{i=2}^{n-1}\left(-\frac{1}{2}\right)^{i-2} r_{i ; f}^{(n)}\right) \\
& \times\left(\begin{array}{cc}
\frac{25}{9} & \frac{5}{7} \\
\frac{5}{7} & \frac{4}{3}
\end{array}\right)^{-1}\left(\sum_{i=2}^{n-1}\left(\frac{4}{5}\right)^{i-2} r_{i ; f}^{(n)}\left(-\frac{1}{2}\right)^{i-2} r_{i ; f}^{(n)}\right) \\
\simeq & n\left(r_{1 ; f f}^{(n)}\right)^{2}+(1.436) n\left[\sum_{i=2}^{n-1}\left(\frac{4}{5}\right)^{i} r_{i ; f}^{(n)}\right]^{2}+(19.600) n\left[\sum_{i=2}^{n-1}\left(-\frac{1}{2}\right)^{i} r_{i ; f}^{(n)}\right]^{2} \\
& -(3.937) n\left[\sum_{i=2}^{n-1}\left(\frac{4}{5}\right)^{i} r_{i ; f}^{(n)}\right]\left[\sum_{i=2}^{n-1}\left(-\frac{1}{2}\right)^{i} r_{i ; f}^{(n)}\right] .
\end{aligned}
$$

If $\operatorname{ARMA}(3,3)[$ or $\operatorname{ARMA}(3,2), \operatorname{ARMA}(3,1), \operatorname{ARMA}(1,3), \ldots]$ alternatives are to be considered, (4.5) has to be modified to

$$
\begin{aligned}
& n\left(r_{1 ; f}^{(n)}\right)^{2}+n\left(r_{2 ; f}^{(n)}\right)^{2}+(2.243) n\left[\sum_{i=3}^{n-1}\left(\frac{4}{5}\right)^{i} r_{i ; f}^{(n)}\right]^{2}+(78.400) n\left[\sum_{i=3}^{n-1}\left(-\frac{1}{2}\right)^{i} r_{i ; f}^{(n)}\right]^{2} \\
& \quad+(9.844) n\left[\sum_{i=3}^{n-1}\left(\frac{4}{5}\right)^{i} r_{i ; f}^{(n)}\right]\left[\sum_{i=3}^{n-1}\left(-\frac{1}{2}\right)^{i} r_{i ; f}^{(n)}\right]
\end{aligned}
$$

\section{Comparison with existing procedures: Concluding remarks.}

5.1. A brief overview of some existing procedures. Tests of time series models adequacy have been extensively studied, and a general survey on the literature on this subject is certainly not possible here. A common feature of the most frequently used procedures is that they do not pay much attention either to the particular model to be tested or to any specific type of alternative. The typical approach consists indeed in computing residuals from the model to be tested, then applying to those residuals some existing test for white noise. Classical examples are the Durbin-Watson test, its higher-order generalizations [Evans and King (1985)] or the popular Ljung-Box-Pierce portmanteau test [Box and Pierce (1970); Ljung and Box (1978)].

More recently, attempts have been made to bring some optimality considerations into the problem. Several authors [see, e.g., Hosking (1978)] have characterized some restricted alternatives against which existing procedures-such as Quenouille's or Box and Pierce's-achieve optimality. A more direct approach has been undertaken by some others, who derive optimal tests for some specific situations [mostly, Gaussian Lagrange multiplier tests-see Godfrey (1979), Hosking (1980), Poskitt and Tremayne (1980); multivariate results are also available: Hosking (1981) and Poskitt and Tremayne (1982)]. A complete result for Gaussian AR(1) series has been obtained in Dufour and King (1986), where 
locally best invariant tests are shown to rely on exponentially weighted sums of sample autocorrelations-a test statistic which is a parametric first-order Gaussian version of our asymptotically sufficient statistic (3.9).

Whereas existing parametric procedures thus either belong to the category of pure significance tests or address restricted classes of Gaussian alternatives, our weighted rank portmanteau tests are providing a locally asymptotically optimal solution to the problem of testing for time series models adequacy in its most general form. Moreover, the proposed solution enjoys all the additional advantages of nonparametric procedures: it is robust and distribution-free.

5.2. Efficiency comparisons. A quantitative comparison of the asymptotic performances of our nonparametric tests with those of existing parametric ones can be achieved through the computation of asymptotic relative efficiencies, whereas small-sample comparisons require extensive Monte Carlo experiments. We therefore limit ourselves to asymptotic considerations; a study of small-sample situations is the purpose of an ongoing research [see Hallin and Mélard (1987) for some results]. Two particular problems, for which an "optimal" parametric Gaussian procedure is available, are considered in some detail: (a) testing the coefficient of an AR(1) model and (b) testing for additional AR or MA roots. For a comparative discussion of weighted rank-based and classical portmanteau tests, we also refer to the end of Section 4.

(a) Testing the coefficient of an AR(1) model. Seven procedures are considered for testing $H_{0}^{(n)}: \rho=\rho_{0},\left|\rho_{0}\right|<1$, against $H_{1}^{(n)}: \rho \neq \rho_{0}$ in the AR(1) model $X_{t}-\rho X_{t-1}=\varepsilon_{t}$. All correlation coefficients are computed from the residuals $Z_{t}^{(n)}=X_{t}^{(n)}-\rho_{0} X_{t-1}^{(n)}$ or from their ranks $R_{t}^{(n)}$. For the sake of convenience, all of the following tests have been given an asymptotic 1 degree of freedom chi-square form. The noncentrality parameters (5.2), (5.4), (5.6), (5.8) and (5.10) are those of the asymptotic noncentral chi-square distributions of the test statistics under the local sequence of alternatives associated with $X_{t}-\left(\rho_{0}+n^{-1 / 2} d\right) X_{t-1}=\varepsilon_{t}$, where $\varepsilon_{t}$ has density $f(\cdot) \cdot \chi_{1-\alpha}^{2}$ stands for the $(1-\alpha)$-quantile of the chi-square distribution with 1 degree of freedom.

(i) Two-sided first-order sample autocorrelation test, two-sided DurbinWatson test, von Neumann's ratio test, Ljung-Box-Pierce test of order 1 (all these tests are asymptotically equivalent):

$$
\text { reject } H_{0}^{(n)} \text { if }(n-1)\left(r_{1}^{(n)}\right)^{2}>\chi_{1-\alpha}^{2},
$$

noncentrality parameter: $d^{2} / 2$.

(ii) Two-sided Dufour and King test: reject $H_{0}^{(n)}$ if

$$
\left(1-\rho_{0}^{2}\right)\left[\sum_{i=1}^{n-1}(n-i)^{1 / 2} \rho_{0}^{i-1} r_{i}^{(n)}\right]^{2} /\left(1-\rho_{0}^{2(n-1)}\right)>\chi_{1-\alpha}^{2},
$$

$$
\text { noncentrality parameter: } d^{2} / 2\left(1-\rho_{0}^{2}\right) \text {. }
$$


(iii)-(v) van der Waerden, Wilcoxon and Laplace (weighted) rank portmanteau tests: reject $H_{0}^{(n)}$ if

$$
\left(1-\rho_{0}^{2}\right)\left[\sum_{i=1}^{n-1}(n-i)^{1 / 2} \rho_{0}^{i-1} r_{i ; g}^{(n)}\right]^{2} /\left(1-\rho_{0}^{2(n-1)}\right)>\chi_{1-\alpha}^{2}
$$

noncentrality parameter: $\quad d^{2}\left[\int_{0}^{1} \phi_{g}\left(G^{-1}(u)\right) \phi_{f}\left(F^{-1}(u)\right) d u\right.$

$$
\left.\times \int_{0}^{1} G^{-1}(v) F^{-1}(v) d v\right]^{2} / 2\left(1-\rho_{0}^{2}\right) I(g) ;
$$

here $g, G, \phi_{g}$ and $I(g)$ denote the standard normal, logistic and double exponential densities, distribution functions, scores and Fisher information, respectively.

(vi) Spearman (weighted) rank portmanteau test: reject $H_{0}^{(n)}$ if

$$
\left(1-\rho_{0}^{2}\right)\left[\sum_{i=1}^{n-1}(n-i)^{1 / 2} \rho_{0}^{i-1} \tilde{r}_{i}^{(n)}\right]^{2} /\left(1-\rho_{0}^{2(n-1)}\right)>\chi_{1-\alpha}^{2}
$$

noncentrality parameter: $144 d^{2}\left[\int_{0}^{1} u \phi\left(F^{-1}(u)\right) d u\right.$

$$
\left.\times \int_{0}^{1} v F^{-1}(v) d v\right]^{2} / 2\left(1-\rho_{0}^{2}\right)
$$

$\tilde{r}_{i}^{(n)}$ stands here [and in (5.9)] for the Spearman-Wald-Wolfowitz rank autocorrelation coefficient; see Hallin and Mélard (1987), Hallin and Puri (1987) and Dufour and Hallin (1987).

(vii) Bartels' (1982) two-sided rank version of von Neumann's ratio test:

$$
\text { reject } H_{0}^{(n)} \text { if }(n-1)\left(\tilde{r}_{1}^{(n)}\right)^{2}>\chi_{1-\alpha}^{2},
$$

$$
\text { noncentrality parameter: } 144 d^{2}\left[\int_{0}^{1} u \phi\left(F^{-1}(u)\right) d u \int_{0}^{1} v F^{-1}(v) d v\right]^{2} / 2 \text {. }
$$

The asymptotic relative efficiencies (ARE) of all these tests with respect to each other can be obtained, under various densities $f$, as the ratios of their respective noncentrality parameters. Table 1 provides some numerical values.

(b) Testing against additional AR or MA roots. The problem considered here is a generalization of the one studied in Hosking (1978); see Remark 4.3 for description. Five procedures are compared here: the Ljung-Box-Pierce parametric portmanteau test (of order $\pi$ if $\pi$ additional roots are to be tested) which is also, asymptotically, the Gaussian likelihood ratio test and four rank-based portmanteau tests (of order $\pi$; in this particular case, they are of the unweighted type). According to Proposition 4.3 (and using the same notation), their asymptotic powers, against contiguous alternatives of the form considered in Remark 4.3 and such that $\sum_{i=1}^{\pi}\left(a_{i}+b_{i}\right)^{2}=d^{2}$, are of the form $1-F_{\pi}\left(\chi_{\pi ; 1-\alpha}^{2} ; \lambda^{2}\right)$, where $\lambda^{2}$ is obtained by letting $\rho_{0}=0$ in the noncentrality parameters (5.4) (for the 
TABLE 1

Asymptotic relative efficiencies of various parametric and nonparametric test procedures for problems $(a)$ and $(b)$ (denoted by superscripts $(a)$ and $(b)$, respectively) under various density types. For each density type, the ARE's are computed with respect to the locally asymptotically optimal test(s) (ARE value of 1$)$.

\begin{tabular}{|c|c|c|c|c|c|c|c|}
\hline \multirow[b]{2}{*}{$\begin{array}{l}\text { Density } \\
\text { type } f\end{array}$} & \multicolumn{2}{|c|}{ Parametric } & \multicolumn{5}{|c|}{ Nonparametric } \\
\hline & $\begin{array}{l}r_{1}^{(n)} \\
-\end{array}$ & $\begin{array}{c}\text { Dufour-King }{ }^{\mathbf{a}} \\
\text { Ljung-Box- }^{\text {Pierce }}{ }^{\mathrm{b}}\end{array}$ & $\begin{array}{c}\text { van der } \\
\text { Waerden }^{\mathrm{a}} \\
\text { van der } \\
\text { Waerden }^{\mathrm{b}}\end{array}$ & $\begin{array}{l}\text { Wilcoxon } \\
\text { Wilcoxon }^{\mathrm{b}}\end{array}$ & $\begin{array}{l}\text { Laplace }^{a} \\
\text { Laplace }^{b}\end{array}$ & $\begin{array}{l}\text { Spearman }^{a} \\
\text { Spearman }^{b}\end{array}$ & Bartels $^{\mathbf{a}}$ \\
\hline Gaussian & $\left(1-\rho_{0}^{2}\right)$ & 1 & 1 & 0.948 & 0.613 & 0.912 & $0.912\left(1-\rho_{0}^{2}\right)$ \\
\hline Logistic & $0.912\left(1-\rho_{0}^{2}\right)$ & 0.912 & 0.954 & 1 & 0.741 & 0.912 & $0.912\left(1-\rho_{0}^{2}\right)$ \\
\hline $\begin{array}{l}\text { Double } \\
\text { exponential }\end{array}$ & $0.500\left(1-\rho_{0}^{2}\right)$ & 0.500 & 0.613 & 0.741 & 1 & 0.613 & $0.613\left(1-\rho_{0}^{2}\right)$ \\
\hline
\end{tabular}

Ljung-Box-Pierce portmanteau test), (5.6) (for the van der Waerden, Wilcoxon and Laplace unweighted portmanteau tests) and (5.8) (for the Spearman unweighted portmanteau test), respectively.

Some numerical values are provided in Table 1.

An inspection of Table 1 reveals the excellent asymptotic performances of rank portmanteau tests: the van der Waerden tests perform uniformly strictly better than the optimal normal-theory tests [viz. the Dufour-King test in problem (a) and the usual Ljung-Box-Pierce portmanteau test in problem (b)] -except of course in the case of Gaussian series, where they perform equally well. The ARE of optimal normal-theory tests with respect to Laplace portmanteau tests can be as low as $\frac{1}{2}$. Note that the Wilcoxon portmanteau tests uniformly dominate the corresponding Spearman-Wald-Wolfowitz procedures. As for the Durbin-Watson and von Neumann ratio tests, their relative performances in problem (a) with respect to optimal rank-based procedures can be arbitrarily bad as $\left|\rho_{0}\right| \rightarrow 1$.

\section{APPENDIX 1}

Difference operators and difference equations. ARMA models characterize stochastic processes as solutions of stochastic difference equations. We briefly review here some basic results on difference operators and difference equations that are used throughout the present paper. These results are univariate constant-coefficient versions of more general results given in Hallin (1986) [see also Miller (1968)].

Denote by $\Theta_{1}, \ldots, \Theta_{p}$ a $p$-tuple of real coefficients with $\Theta_{p} \neq 0$ and by $L$ the lag or backshift operator $\left(L z_{t}=z_{t-1}\right)$. These coefficients characterize a linear difference operator of order $p: \Theta(L)=1+\sum_{i=1}^{p} \Theta_{i} L^{i}$. Such an operator defines difference equations $\Theta(L) \Psi_{t}=\omega_{t}, t \in \mathbb{Z}$, the solutions of which are completely determined by $p$ successive "initial" values.

The Green's function $G_{t}$ associated with the operator $\Theta(L)$ is the solution of the homogeneous equation $\Theta(L) G_{t}=0, t \in \mathbb{Z}$, taking on initial values $G_{0}=1$, $G(-1)=\cdots=G(-p+1)=0$. 
It is easy to see that the set of all solutions of the homogeneous equation $\Theta(L) \Psi_{t}=0, t \in \mathbf{Z}$, constitutes a vector space of dimension $p$. A basis of this vector space is called a fundamental system of solutions. Two fundamental systems are particularly convenient:

(a) Denote by $\lambda_{(1)}, \ldots, \lambda_{(p)}$ the roots of the polynomial $\Theta\left(z^{-1}\right)$, i.e., the solutions of $1+\Theta_{1} z^{-1}+\cdots+\Theta_{p} z^{-p}=0, z \in \mathbb{C}$. Assume that all these roots are of multiplicity 1 . Then $\left\{\lambda_{(1)}^{t}, \ldots, \lambda_{(p)}^{t}\right\}$ constitutes a fundamental system of solutions.

(b) Another fundamental system is provided by the p-tuple of Green's functions $\left\{G_{t}, G_{t+1}, \ldots, G_{t+p-1}\right\}$; see Hallin [(1986), Theorem 1.1].

Using this latter fundamental system, the general solution of $\Theta(L) \Psi_{t}=\omega_{t}$ is

$$
\Psi_{t}=\left\{\begin{array}{rr}
\sum_{u=0}^{t-1} G_{u} \omega_{t-u}+\left(G_{t}, G_{t+1}, \ldots, G_{t+p-1}\right) \mathbf{C}^{-1}\left(\Psi_{0}, \Psi_{-1}, \ldots, \Psi_{-p+1}\right)^{\prime}, \\
t \geq 1, \\
\sum_{u=t}^{-p} G_{u} \omega_{t-u}+\left(G_{t}, G_{t+1}, \ldots, G_{t+p-1}\right) \mathbf{C}^{-1}\left(\Psi_{0}, \Psi_{-1}, \ldots, \Psi_{-p+1}\right)^{\prime}, \\
t \leq-p,
\end{array}\right.
$$

where

$$
\mathbf{C}^{-1}=\left(\begin{array}{cccc}
G_{0} & G_{1} & \cdots & G_{p-1} \\
G_{-1} & G_{0} & & G_{p-2} \\
\vdots & \vdots & \ddots & \vdots \\
G_{-p+1} & G_{-p+2} & \cdots & G_{0}
\end{array}\right)^{-1}=\left(\begin{array}{cccc}
1 & \Theta_{1} & \cdots & \Theta_{p-1} \\
0 & \ddots & \ddots & \vdots \\
\vdots & \ddots & \ddots & \Theta_{1} \\
0 & \cdots & 0 & 1
\end{array}\right) .
$$

[C is the value for $t=0$ of the Casorati matrix associated with the fundamental system $\left\{G_{t}, G_{t+1}, \ldots, G_{t+p-1}\right\}$.]

\section{APPENDIX 2}

Exact and approximate residuals. The exact residuals $\mathbf{Z}^{(n)}=$ $\left(Z_{1}^{(n)}, \ldots, Z_{n}^{(n)}\right)$, where $Z_{t}^{(n)}=[A(L) / B(L)] X_{t}^{(n)}, 1 \leq t \leq n$, cannot be derived from the observed series $\mathbf{X}^{(n)}$ [unless $B(L) \equiv 1$ ]. From Appendix 1 , we have indeed

$$
\begin{aligned}
Z_{t}^{(n)}= & \sum_{u=0}^{t-1} H_{u}\left(1-\sum_{j=1}^{p_{1}} A_{j} L^{j}\right) X_{t-u}^{(n)} \\
& +\left(H_{t}, \ldots, H_{t-q_{1}+1}\right) \mathbf{C}^{-1}\left(Z_{0}^{(n)}, \ldots, Z_{-q_{1}+1}^{(n)}\right)^{\prime}, \quad t>0,
\end{aligned}
$$

where the values of $X_{t}^{(n)},-p_{1}+1 \leq t \leq 0$ and $Z_{t}^{(n)},-q_{1}+1 \leq t \leq 0$ are not available from the observations. Approximate residuals $\hat{Z}_{t}^{(n)}$ can be obtained by putting $X_{0}^{(n)}=X_{-1}^{(n)}=\cdots=X_{-p_{1}+1}^{(n)}=0=Z_{0}^{(n)}=Z_{-1}^{(n)}=\cdots=Z_{-q_{1}+1}^{(n)}$ in (A2.1) or, equivalently, by applying, from the previous initial values, the recursion formula $\hat{Z}_{t}^{(n)}=X_{t}^{(n)}-\sum_{j=1}^{p_{1}} A_{j} X_{t-j}^{(n)}-\sum_{i=1}^{q_{1}} B_{i} \hat{Z}_{t-i}^{(n)}$. More sophisticated 
devices yielding better small-sample properties such as backforecasting can also be used [see, e.g., Box and Jenkins (1970), pages 199-200], but the effect of the chosen initial values is asymptotically negligible. Under assumption (b) (Section 3.1 , the difference $\Delta_{t}^{(n)}=\hat{Z}_{t}^{(n)}-Z_{t}^{(n)}$ is $O_{p}\left(\Lambda^{t}\right)$, as $t \rightarrow \infty$, where $\Lambda<1$ is the modulus of the largest root of $A\left(z^{-1}\right) B\left(z^{-1}\right)=0, z \in \mathbb{C}$. Denote by $\hat{S}^{(n)^{*}}$ the value of the optimal statistic (2.8) when the ranks $R_{t}^{(n)}$ of the exact residuals $Z_{t}^{(n)}$ are replaced by those $\hat{R}_{t}^{(n)}$ of the approximate residuals $\hat{Z}_{t}^{(n)}$. Our objective is to show that, as $n \rightarrow \infty, \hat{S}^{(n)^{*}}$ and $S^{(n)^{*}}$ are asymptotically equivalent [i.e., $\left.n^{1 / 2}\left(\hat{S}^{(n)^{*}}-S^{(n)^{*}}\right)=o_{p}(1)\right]$, so that the results of the paper are not affected if approximate residuals are used. Consider the problem of testing $H^{(n)}(\mathbf{A}, \mathbf{B} ; f)$ against $K^{(n)}(\mathbf{A}, \mathbf{B} ; \gamma, \delta ; f)$. Referring to the proof of Proposition 2.2 (A3.1), we have

$$
\begin{aligned}
& \log L^{(n)}\left(\mathbf{Z}^{(n)}\right)-\log L^{(n)}\left(\hat{\mathbf{Z}}^{(n)}\right) \\
& \quad=\log E\left[\lambda^{(n)}\left(\mathbf{Z}^{(n)}+\Delta^{(n)}, \mathbf{X}_{0}^{(n)}\right)-\lambda^{(n)}\left(\mathbf{Z}^{(n)}, \mathbf{X}_{0}^{(n)}\right) \mid \mathbf{Z}^{(n)}\right] .
\end{aligned}
$$

Carrying on Taylor's expansion of $\lambda^{(n)}\left(\mathbf{Z}^{(n)}+\Delta^{(n)}, \mathbf{X}_{0}^{(n)}\right)$ (with respect to the $\Delta_{t}^{(n)}$ 's), it is easy to see that under $H^{(n)}(\mathbf{A}, \mathbf{B} ; f)$, the difference (A2.2) is $o_{p}(1)$ as $n \rightarrow \infty$ [the arguments involved are essentially the same as in the proof of Proposition 2.2 with the additional fact that quantities of the form $a_{i} \Delta_{t-i}^{(n)}$ are $\left.O_{p}\left(\Lambda^{t}\right)\right]$. Denote by $\hat{H}_{f}^{(n)}(\mathbf{A}, \mathbf{B} ; f)$ the hypothesis under which $\left(\hat{Z}_{1}^{(n)}, \ldots, \hat{Z}_{n}^{(n)}\right)$ are independent and identically distributed with density $f(\cdot) . \hat{H}_{f}^{(n)}(\cdots)$ and $H_{f}^{(n)}(\cdots)$ are contiguous [because the sequences of the distributions of $\sum_{t=1}^{n} \log \left(f\left(\hat{Z}_{t}^{(n)}\right) / f\left(Z_{t}^{(n)}\right)\right)$ are relatively compact under both $H_{f}^{(n)}(\cdots)$ and $\hat{H}_{f}^{(n)}(\cdots)$; Le Cam (1960)]. Taking (A3.9) into account, it follows that

$$
\begin{aligned}
n^{1 / 2}\left(\hat{S}^{(n)^{*}}-S^{(n)^{*}}\right)= & {\left[\log L^{(n)}\left(\hat{\mathbf{Z}}^{(n)}\right)-\log L^{(n)}\left(\mathbf{Z}^{(n)}\right)\right] } \\
& \times\left[\|\mathbf{a}+\mathbf{b}\|^{2} \boldsymbol{\sigma}^{2} I(f)\right]^{-1 / 2}+o_{p}(1) \\
= & o_{p}(1) .
\end{aligned}
$$

(A2.3) holds under $H^{(n)}(\mathbf{A}, \mathbf{B} ; f)$ and, because of Le Cam's third lemma, it also holds under $K^{(n)}(\mathbf{A}, \mathbf{B} ; \gamma, \delta ; f)$, which establishes the desired result.

\section{APPENDIX 3}

\section{Proofs.}

Proof of Proposition 2.2. The proof mainly consists in decomposing $\log L^{(n)}\left(\mathbf{X}^{(n)}\right)$ into

(A3.1) $\log L^{(n)}\left(\mathbf{X}^{(n)}\right)=\mathscr{L}_{\kappa}^{(n)}\left(\mathbf{X}^{(n)}\right)-\frac{1}{2} \sum_{i=1}^{\infty}\left(a_{i}+b_{i}\right)^{2} \sigma^{2} I(f)+R_{\kappa}^{(n)}+o_{p}$,

where $\mathscr{L}_{\kappa}^{(n)}\left(\mathbf{X}^{(n)}\right)=n^{-1 / 2} \sum_{t=\kappa+1}^{n} \phi\left(X_{t}\right) \sum_{i=1}^{\kappa}\left(a_{i}+b_{i}\right) X_{t-i}$ is asymptotically normal with mean 0 and variance $\sum_{i=1}^{\kappa}\left(a_{i}+b_{i}\right)^{2} \sigma^{2} I(f)$ [this follows from Proposition 3.1 in Hallin, Ingenbleek and Puri (1985)], and $R_{\kappa}^{(n)}$ converges to zero in 
probability [under $H_{0 ; f}^{(n)}$ ] as $\kappa \rightarrow \infty$, uniformly with respect to $n$. Since the asymptotic distribution of $\mathscr{L}_{\kappa}^{(n)}\left(\mathbf{X}^{(n)}\right)$ clearly converges, as $\kappa \rightarrow \infty$, to the normal distribution with mean zero and variance $\sum_{i=1}^{\infty}\left(a_{i}+b_{i}\right)^{2} \sigma^{2} I(f)$, the asymptotic normality of $\log L^{(n)}\left(\mathbf{X}^{(n)}\right)$ follows from Theorem 2.1. Contiguity then immediately stems from Le Cam's first lemma [Hájek and Šidák (1967), page 204].

A decomposition of the desired (A3.1) form is obtained by considering a Taylor expansion of the log-likelihood ratio. In order to avoid purposeless intricate computations, we are treating here in some detail the case where $\mathbf{b}=(0,0, \ldots)$. (2.1) then takes the form of an $\operatorname{AR}(\infty)$ model. The general case can be treated along the same lines by introducing the $\mathrm{AR}(\infty)$ form of (2.1), $X_{t}-\sum_{i=1}^{\infty} d_{i}^{(n)} X_{t-i}=\varepsilon_{t}$, then showing that it is asymptotically equivalent with a model of the form $X_{t}-n^{-1 / 2} \sum_{i=1}^{\infty}\left(a_{i}+b_{i}\right) X_{t-i}=\varepsilon_{t}$ [see Hallin, Ingenbleek and Puri (1985), Appendix 2b].

Denote by $\mathbf{X}_{0}^{(n)}=\left(X_{0}^{(n)}, X_{-1}^{(n)}, \ldots\right)$ the stationary solution (for $\left.t \leq 0\right)$ of $(2.1)$ and by $\mathbf{X}^{(n)}$ an $n$-tuple of i.i.d. variables with common density $f(x)$, independent from $\mathrm{X}^{(n)}$. Under $H_{0 ; f}^{(n)}$, the log-likelihood ratio can be expressed as

$$
\log L^{(n)}\left(\mathbf{X}^{(n)}\right)=\log E\left[\lambda^{(n)}\left(\mathbf{X}^{(n)}, \mathbf{X}_{0}^{(n)}\right) \mid \mathbf{X}^{(n)}\right],
$$

with $\lambda^{(n)}\left(\mathbf{X}^{(n)}, \mathbf{X}_{0}^{(n)}\right)=\prod_{t=1}^{n} f\left(X_{t}^{(n)}-n^{-1 / 2} \sum_{i=1}^{\infty} a_{i} X_{t-i}^{(n)}\right) / f\left(X_{t}^{(n)}\right)$. Expanding $\log \lambda^{(n)}\left(\mathbf{X}^{(n)}, \mathbf{X}_{0}^{(n)}\right)$ yields

$$
\begin{aligned}
\log \lambda^{(n)}\left(\mathbf{X}^{(n)}, \mathbf{X}_{0}^{(n)}\right)= & n^{-1 / 2} \sum_{t=1}^{n} \phi\left(X_{t}^{(n)}\right) \sum_{i=1}^{\infty} a_{i} X_{t-i}^{(n)} \\
& -(2 n)^{-1} \sum_{t=1}^{n} \phi^{\prime}\left(X_{t}^{(n)}-\theta n^{-1 / 2} \sum_{i=1}^{\infty} a_{i} X_{t-i}^{(n)}\right)\left(\sum_{i=1}^{\infty} a_{i} X_{t-i}^{(n)}\right)^{2},
\end{aligned}
$$

with $\theta=\theta\left(\mathbf{X}^{(n)}, \mathbf{X}_{0}^{(n)}\right) \in[0,1]$. First let us consider the second-order term in (A3.3). Since $\phi^{\prime}$ is Lipschitzian,

$$
\begin{aligned}
& n^{-1}\left|\sum_{t=1}^{n}\left[\phi^{\prime}\left(X_{t}^{(n)}-\theta n^{-1 / 2} \sum_{i=1}^{\infty} a_{i} X_{t-i}^{(n)}\right)-\phi^{\prime}\left(X_{t}^{(n)}\right)\right]\left[\sum_{i=1}^{\infty} a_{i} X_{t-i}^{(n)}\right]^{2}\right| \\
& \quad \leq n^{-3 / 2} K \sum_{t=1}^{n}\left|\sum_{i=1}^{\infty} a_{i} X_{t-i}^{(n)}\right|^{3} .
\end{aligned}
$$

But, under $H_{0 ; f}^{(n)}, X_{t}^{(n)}$ has finite moments of order 6, both for $t>0$ as for $t \leq 0$. Since $\sum_{i=1}^{\infty}\left|a_{i}\right|<\infty$, the variance of (A3.4) is $O\left(n^{-1}\right)$ and the second-order term in (A3.3) reduces to

$$
\begin{aligned}
-(2 n)^{-1} \sum_{t=1}^{n} \phi^{\prime}\left(X_{t}^{(n)}\right)\left(\sum_{i=1}^{\infty} a_{i} X_{t-i}^{(n)}\right)^{2}+o_{p} \\
=-(2 n)^{-1} \sum_{t=1}^{n} \phi^{\prime}\left(X_{t}^{(n)}\right)\left(\sum_{i=1}^{\kappa} a_{i} X_{t-i}^{(n)}\right)^{2}-(2 n)^{-1} \sum_{t=1}^{n} \phi^{\prime}\left(X_{t}^{(n)}\right) \\
\times\left[2 \sum_{i=1}^{\kappa} a_{i} X_{t-i}^{(n)} \sum_{i=\kappa+1}^{\infty} a_{i} X_{t-i}^{(n)}+\left(\sum_{i=\kappa+1}^{\infty} a_{i} X_{t-i}^{(n)}\right)^{2}\right]+o_{p} .
\end{aligned}
$$


The first sum on the right-hand side of (A3.5) is a sum of identically distributed $\kappa$-dependent terms; it therefore converges, as $n \rightarrow \infty$, to $(t>\kappa)$

$$
-\frac{1}{2} E\left[\phi^{\prime}\left(X_{t}^{(n)}\right)\left(\sum_{i=1}^{\kappa} a_{i} X_{t-i}^{(n)}\right)^{2}\right]=-\frac{1}{2} \sigma^{2} I(f) \sum_{i=1}^{\kappa} a_{i}^{2} .
$$

As for the remainder term, let us show that it converges to zero, as $\kappa \rightarrow \infty$, uniformly with respect to $n$. Taking into account the fact that $X_{t}^{(n)}$ has finite fourth-order moments, as well for $t>0$ as for $t \leq 0$, we obtain

$$
\begin{aligned}
& E\left\{(2 n)^{-1} \sum_{t=1}^{n} \phi^{\prime}\left(X_{t}^{(n)}\right)\left[\sum_{i=\kappa+1}^{\infty} a_{i} X_{t-i}^{(n)}\right]^{2}\right\}^{2} \\
&=\frac{1}{4 n^{2}}\left\{\sum_{t=1}^{n} E\left[\left(\phi^{\prime}\left(X_{t}^{(n)}\right)\right)^{2}\right] E\left[\left(\sum_{i=\kappa+1}^{\infty} a_{i} X_{t-i}^{(n)}\right)^{4}\right]\right. \\
&\left.\quad+2 \sum_{1 \leq s<t \leq n} E\left[\phi^{\prime}\left(X_{t}^{(n)}\right)\right] E\left[\phi^{\prime}\left(X_{s}^{(n)}\right)\left(\sum_{i=\kappa+1}^{\infty} a_{i} X_{t-i}^{(n)}\right)^{2}\left(\sum_{i=\kappa+1}^{\infty} a_{i} X_{s-i}^{(n)}\right)^{2}\right]\right\} \\
& \leq \frac{1}{4 n} E\left[\left(\phi^{\prime}\left(X_{1}^{(n)}\right)\right)^{2}\right] K\left(\sum_{i=0}^{\infty}\left|a_{\kappa+1+i}\right|\right)^{4} \\
& \quad+\frac{1}{4} I(f) E\left[\left(\phi^{\prime}\left(X_{1}^{(n)}\right)\right)^{2}\right]^{1 / 2} K\left(\sum_{i=0}^{\infty}\left|a_{\kappa+1+i}\right|\right)^{4} \\
& \leq K^{\prime}\left(\sum_{i=\kappa}^{\infty}\left|a_{i}\right|\right)^{4} .
\end{aligned}
$$

In a very similar way,

$$
E\left\{(2 n)^{-1} \sum_{t=1}^{n} \phi^{\prime}\left(X_{t}^{(n)}\right)\left(\sum_{i=1}^{\kappa} a_{i} X_{t-i}^{(n)}\right)\left(\sum_{i=\kappa+1}^{\infty} a_{i} X_{t-i}^{(n)}\right)\right\}^{2} \leq K^{\prime \prime}\left(\sum_{i=\kappa}^{\infty}\left|a_{i}\right|\right)^{4} ;
$$

the two constants $K^{\prime}$ and $K^{\prime \prime}$ do not depend on $n$. The right-hand side of (A3.5) therefore satisfies the assumptions of Theorem 2.1 in the particular case where the limit distribution is degenerate $\left[F(z)=1\right.$ for $z \geq-\frac{1}{2} \sum_{i=1}^{\infty} a_{i}^{2} \sigma^{2} I(f), F(z)=0$ elsewhere] and the second-order term in (A3.3) converges in probability to $-\frac{1}{2} \sum_{i=1}^{\infty} a_{i}^{2} \sigma^{2} I(f)$ as $n \rightarrow \infty$. (A3.3) consequently takes the form

$$
\begin{aligned}
\log \lambda^{(n)}\left(\mathbf{X}^{(n)}, \mathbf{X}_{0}^{(n)}\right)= & n^{-1 / 2} \sum_{t=1}^{n} \phi\left(X_{t}^{(n)}\right) \sum_{i=1}^{\infty} a_{i} X_{t-i}^{(n)}-\frac{1}{2} \sum_{i=1}^{\infty} a_{i}^{2} \sigma^{2} I(f)+o_{p} \\
= & \mathscr{L}_{\kappa}^{(n)}\left(\mathbf{X}^{(n)}\right)+n^{-1 / 2} \sum_{t=1}^{n} \phi\left(X_{t}^{(n)}\right) \sum_{i=\kappa+1}^{\infty} a_{i} X_{t-i}^{(n)} \\
& -\frac{1}{2} \sum_{i=1}^{\infty} a_{i}^{2} \sigma^{2} I(f)+o_{p} .
\end{aligned}
$$


Because $E\left[\phi\left(X_{t}^{(n)}\right) \phi\left(X_{s}^{(n)}\right)\right]=\delta_{t s} I(f), t, s=1, \ldots, n$, we have

$$
\begin{aligned}
& E\left\{n^{-1 / 2} \sum_{t=1}^{n} \phi\left(X_{t}^{(n)}\right) \sum_{i=\kappa+1}^{\infty} a_{i} X_{t-i}^{(n)}\right\}^{2} \\
& =\frac{1}{n} \sum_{t=1}^{n} E\left[\left(\phi\left(X_{t}^{(n)}\right)\right)^{2}\right] E\left[\left(\sum_{i=\kappa+1}^{\infty} a_{i} X_{t-i}^{(n)}\right)^{2}\right] \leq K^{\prime}\left(\sum_{i=\kappa}^{\infty}\left|a_{i}\right|\right)^{2} ;
\end{aligned}
$$

the latter quantity again converges to zero as $\kappa \rightarrow \infty$, independently of $n$. Going back to (A3.2),

$$
\begin{aligned}
\log L^{(n)}\left(\mathbf{X}^{(n)}\right)= & \mathscr{L}_{\kappa}^{(n)}\left(\mathbf{X}^{(n)}\right)-\frac{1}{2} \sum_{i=1}^{\infty} a_{i}^{2} \sigma^{2} I(f) \\
& +\log E\left[\exp \left\{n^{-1 / 2} \sum_{t=1}^{n} \phi\left(X_{t}^{(n)}\right) \sum_{i=\kappa+1}^{\infty} a_{i} X_{t-i}^{(n)}\right\} \mid \mathbf{X}^{(n)}\right]+o_{p}
\end{aligned}
$$

which is of the required form (A3.1). In the general case, $\sum_{i=1}^{\infty} a_{i}^{2}$ has to be replaced with $\sum_{i=1}^{\infty}\left(a_{i}+b_{i}\right)^{2}$.

Proof of Proposition 2.3. Consider the $\log$-likelihood ratio $\log L_{\kappa}^{(n)}\left(\mathbf{X}^{(n)}\right)$ for $H_{0 ;}^{(n)}$ against the $\kappa$ th-order "truncation" $K_{\kappa}^{(n)}$ of $K^{(n)}(\mathbf{a}, \mathbf{b} ; f), \kappa \geq p$. We know from Hallin, Ingenbleek and Puri (1985) that the joint distribution of $\left(\log L_{\kappa}^{(n)}, n^{1 / 2}\left(S^{(n)}-m^{(n)}\right)\right)^{\prime}$ is asymptotically normal (under $\left.H_{0 ; f}^{(n)}\right)$, with mean $\left(-\frac{1}{2} \sum_{i=1}^{\kappa}\left(a_{i}+b_{i}\right)^{2} \sigma^{2} I(f), 0\right)^{\prime}$ and covariance matrix

$$
\left(\begin{array}{cc}
\sum_{i=1}^{\kappa}\left(a_{i}+b_{i}\right)^{2} \sigma^{2} I(f) & \cdot \\
\sum_{i=1}^{p}\left(a_{i}+b_{i}\right) C_{i} & V^{2}
\end{array}\right) .
$$

Now the decomposition we obtained in (A3.1) is, up to $o_{p}$ terms, of the form

$$
\text { (A3.6) } \log L^{(n)}\left(\mathbf{X}^{(n)}\right)=\log L_{\kappa}^{(n)}\left(\mathbf{X}^{(n)}\right)+R_{\kappa}^{(n)}-\frac{1}{2} \sum_{\kappa+1}^{\infty}\left(a_{i}+b_{i}\right)^{2} \sigma^{2} I(f) \text {. }
$$

Since $V^{2}$ is finite and does not depend on $n$ or on $\kappa$, Theorem 2.1 can be applied again to an arbitrary linear combination of $\log L^{(n)}\left(\mathbf{X}^{(n)}\right)$ and $n^{1 / 2}\left(S^{(n)}-m^{(n)}\right)$. It follows that the joint distribution of $\log L^{(n)}$ and $n^{1 / 2}\left(S^{(n)}-m^{(n)}\right)$ is asymptotically normal, with mean $\left(-\frac{1}{2} \sum_{i=1}^{\infty}\left(a_{i}+b_{i}\right)^{2} \sigma^{2} I(f), 0\right)^{\prime}$ and the same covariance matrix as before, except for the first diagonal element, which is now $\sum_{i=1}^{\infty}\left(a_{i}+b_{i}\right)^{2} \sigma^{2} I(f)$. Proposition 2.3 then straightforwardly follows from Le Cam's third lemma.

Proof of Proposition 2.4. (i) The proof of part (i) of the proposition readily follows from Le Cam's third lemma by applying Theorem 2.1 and Proposition 3.2 in Hallin, Ingenbleek and Puri (1987) to an arbitrary linear 
combination of $\log L^{(n)}\left(\mathbf{X}^{(n)}\right)$ and $n^{1 / 2} S_{\kappa}^{(n)^{*}}$, where

$$
\begin{aligned}
n^{1 / 2} S_{\kappa}^{(n)^{*}}= & \frac{n^{1 / 2}}{\|\mathbf{a}+\mathbf{b}\|} S^{(n)^{*}}\left[\sum_{i=1}^{n-1}\left(a_{i}+b_{i}\right)^{2}\right]^{1 / 2} \\
& -\sum_{i=\kappa+1}^{n-1}(n-i)^{1 / 2}\left(a_{i}+b_{i}\right) \frac{r_{i ; i}^{(n)}}{\|\mathbf{a}+\mathbf{b}\|} .
\end{aligned}
$$

(ii) Write $K^{(n)}$ for $K^{(n)}(\mathbf{a}, \mathbf{b} ; f)$. It follows from (2.9) that the asymptotic power of the test based on $S^{(n)^{*}}$ for $H_{0}^{(n)}$ against $K^{(n)}$ is $1-\Phi\left(k_{1-\alpha}-\right.$ $\left.\|\mathbf{a}+\mathbf{b}\|\left[\sigma^{2} I(f)\right]^{1 / 2}\right)$, where $\Phi$ denotes the standard normal distribution function. Using classical notation ( Section 3.2), let $\beta(\alpha, H, K)$ be the envelope power function for testing $H$ against $K$ at level $\alpha$. Then $\beta\left(\alpha, H_{0 ; f}^{(n)}, K^{(n)}\right)$ is the power reached by the likelihood ratio test. Using Proposition 2.2, we obtain

$$
\lim _{n \rightarrow \infty} \beta\left(\alpha, H_{0 ; j}^{(n)}, K^{(n)}\right)=1-\Phi\left(k_{1-\alpha}-\|\mathbf{a}+\mathbf{b}\|\left[\sigma^{2} I(f)\right]^{1 / 2}\right) .
$$

Since $\beta\left(\alpha, H_{0 ; f}^{(n)}, K^{(n)}\right) \geq \beta\left(\alpha, H_{0}^{(n)}, K^{(n)}\right)$ for every $n$, we have

Therefore,

$$
\lim _{n \rightarrow \infty} \beta\left(\alpha, H_{0 ; f}^{(n)}, K^{(n)}\right) \geq \underset{n \rightarrow \infty}{\limsup } \beta\left(\alpha, H_{0}^{(n)}, K^{(n)}\right) .
$$

$$
\begin{aligned}
\limsup _{n \rightarrow \infty} \beta\left(\alpha, H_{0}^{(n)}, K^{(n)}\right) & \geq 1-\Phi\left(k_{1-\alpha}-\|\mathbf{a}+\mathbf{b}\|\left[\sigma^{2} I(f)\right]^{1 / 2}\right) \\
& =\lim _{n \rightarrow \infty} \beta\left(\alpha, H_{0 ; f}^{(n)}, K^{(n)}\right),
\end{aligned}
$$

which implies that $\lim _{n \rightarrow \infty} \beta\left(\alpha, H_{0}^{(n)}, K^{(n)}\right)$ exists and equals the asymptotic power of (2.9).

Proof of Proposition 3.2. Let $l_{0 ; f}^{(n)}\left(\mathbf{X}^{(n)}\right)=\prod_{t=1}^{n} f\left(Z_{t}^{(n)}\right)$. The objective is to show that there exist $\mathbf{T}_{\Psi ; f}^{(n)}$-measurable variables $h_{\gamma, \delta}^{(n)}$, such that the functions

$$
\bar{l}_{\gamma, \delta}^{(n)}=l_{0 ; f}^{(n)} h_{\gamma, \delta}^{(n)}
$$

are densities for all $(\gamma, \delta) \in \mathscr{D}(d)$ and $n$ ( $n$ sufficiently large), and satisfy

$$
\lim _{n \rightarrow \infty} \sup _{\mathscr{D}(d)}\left\|l_{\gamma, \delta}^{(n)}-\bar{l}_{\gamma, \delta}^{(n)}\right\|_{L_{1}}=0,
$$

where $\|p-q\|_{L_{1}}$ is the $L_{1}$-distance $\int|p-q| d \mu$ for densities defined with respect to the $\sigma$-finite measure $\mu$.

Consider the optimal rank statistic $S_{\mathbf{a}, \mathbf{b}}^{(n)^{*}}$ we introduced in Propositions 2.4 and 3.1. It follows from the proof of Proposition 2.4 that asymptotically the joint distribution of $\log l_{\gamma, \delta}^{(n)} / l_{0 ; j}^{(n)}$ and $n^{1 / 2} S_{\mathbf{a}, \mathbf{b}}^{(n)^{*}}$ is normal, with covariance matrix

$$
\left(\begin{array}{cc}
\|\mathbf{a}+\mathbf{b}\|^{2} \boldsymbol{\sigma}^{2} I(f) & \cdot \\
\|\mathbf{a}+\mathbf{b}\|\left[\boldsymbol{\sigma}^{2} I(f)\right]^{1 / 2} & 1
\end{array}\right),
$$

from which we deduce that

$$
\log \left[L_{\gamma, 8}^{(n)} / l_{0 ; f}^{(n)}\right]+\frac{1}{2}\|\mathbf{a}+\mathbf{b}\|^{2} \sigma^{2} I(f)-n^{1 / 2}\|\mathbf{a}+\mathbf{b}\|\left[\sigma^{2} I(f)\right]^{1 / 2} S_{\mathbf{a}, \mathbf{b}}^{(n)^{*}}
$$


converges to zero, in probability, under $H^{(n)}(\mathbf{A}, \mathbf{B} ; f)$. Moreover, this convergence is uniform with respect to the $\gamma_{i}$ 's and $\delta_{i}$ 's, $(\gamma, \delta) \in \mathscr{D}(d)$. Hence writing $s_{\mathbf{a}, \mathbf{b}}^{(n)^{*}}$ for $n^{1 / 2}\|\mathbf{a}+\mathbf{b}\|\left[\sigma^{2} I(f)\right]^{1 / 2} S_{\mathbf{a}, \mathbf{b}}^{(n)^{*}}$ [with $\mathbf{a}$ and $\mathbf{b}$ given in (3.2)], we have

$$
\lim _{n \rightarrow \infty} \sup _{\mathscr{D}(d)} P_{l_{0}^{(n)}}\left[\left|\left(l_{\gamma, 8}^{(n)} / l_{0 ; f}^{(n)}\right)-\exp \left(s_{\mathbf{a}, \mathbf{b}}^{(n)^{*}}-\frac{1}{2}\|\mathbf{a}+\mathbf{b}\|^{2} \mathbf{\sigma}^{2} I(f)\right)\right|>\varepsilon\right]=0
$$

for any $\varepsilon>0$, which is equivalent to Hájek and Šidák's equation (10) [(1967), page 246].

Defining $h_{\gamma, \delta}^{(n)}$ as

$$
h_{\gamma, \mathbf{8}}^{(n)}= \begin{cases}B_{\gamma, \mathbf{8}}^{(n)} \exp \left[s_{\mathbf{a}, \mathbf{b}}^{(n)}-\frac{1}{2}\|\mathbf{a}+\mathbf{b}\|^{2} \sigma^{2} I(f)\right], & \text { if }\left|s_{\mathbf{a}, \mathbf{b}}^{(n)^{*}}\right| \leq C^{(n)}, \\ 0, & \text { if }\left|s_{\mathbf{a}, \mathbf{b}}^{(n)^{*}}\right|>C^{(n)}\end{cases}
$$

where $B_{\gamma, 8}^{(n)}$ is a normalizing constant such that (A3.7) is a probability density function, and reproducing Hájek and Šidák's proof of Theorem 7.1.1 [(1967), pages $247-248$, including their definitions of constants $\left.C^{(n)}\right]$ leads to the conclusion that (A3.7) and (A3.8) are satisfied.

In order to complete the proof, it remains to show that the variables $s_{\mathbf{a}, \mathbf{b}}^{(n)}$, hence the $h_{\gamma, 8}^{(n)}$ 's are $\mathbf{T}_{\Psi ; f}^{(n)}$-measurable. Notice therefore that, for $t \geq p_{2}-p_{1}+1$ and $t^{\prime} \geq q_{2}-q_{1}+1, \quad a_{t}=\sum_{j=1}^{p_{2}} \gamma_{j} G_{t-j}$ and $b_{t^{\prime}}=\sum_{j=1}^{q_{2}} \delta_{j} H_{t^{\prime}-j}$. Now $\left\{G_{t-1}, \ldots, G_{t-p_{1}}\right\}$ and $\left\{H_{t-1}, \ldots, H_{t-q_{1}}\right\}$ constitute fundamental systems of solutions of $A(L) \Psi_{t}=0, t \in \mathbb{Z}$, and $B(L) \Psi_{t}=0, t \in \mathbb{Z}$, respectively (see Appendix $1)$, and $G_{t-p_{1}-1}, \ldots, G_{t-p_{2}}$ and $H_{t-q_{1}-1}, \ldots, H_{t-q_{2}}$ are solutions of the same equations, respectively. Hence, for $t \geq \pi+1, A(L) a_{t}=0=B(L) b_{t}$, from which we deduce that $A(L) B(L)\left(a_{t}+b_{t}\right)=0, t \geq \pi+1$. It follows that

(A3.10) $\left(a_{i}+b_{i}\right)=\left(k_{1}, \ldots, k_{p_{1}+q_{1}}\right)\left(\Psi_{i}^{(1)}, \ldots, \Psi_{i}^{\left(p_{1}+q_{1}\right)}\right)^{\prime}, \quad i \geq \pi+1$,

where the constants $k_{1}, \ldots, k_{p_{1}+q_{1}}$ depend on $\gamma$ and $\delta$ only (for given $\Psi_{t}^{(j)}$ 's). Going back to the definition of $s_{\mathbf{a}+\mathbf{b}}^{(n) *}$, we finally obtain

$$
s_{\mathbf{a}+\mathbf{b}}^{(n)^{*}}=n^{1 / 2} \frac{\|\mathbf{a}+\mathbf{b}\|\left[\sigma^{2} I(f)\right]^{1 / 2}}{\left[\sum_{i=1}^{n-1}\left(a_{i}+b_{i}\right)^{2}\right]^{1 / 2}}\left(\left(a_{1}+b_{1}\right), \ldots,\left(a_{\pi}+b_{\pi}\right), k_{1}, \ldots, k_{p_{1}+q_{1}}\right) \mathbf{T}_{\Psi ; f}^{(n)},
$$

which completes the proof.

Proof of Proposition 3.3. Let $\kappa>\pi$ and consider an arbitrary linear combination of $\log L^{(n)}\left(\mathbf{Z}^{(n)}\right), n^{1 / 2} r_{1 ; f}^{(n)}, \ldots, n^{1 / 2} r_{\pi ; f}^{(n)}, n^{1 / 2} \sum_{i=\pi+1}^{\kappa} \Psi_{i}^{(1)} r_{i ; f}^{(n)}, \ldots$, and $n^{1 / 2} \sum_{i=\pi+1}^{\kappa} \Psi_{i}^{\left(p_{1}+q_{1}\right)} r_{i ; f}^{(n)}$. The asymptotic normality of this linear combination can easily be established along the lines of Proposition 3.1 in Hallin, Ingenbleek and Puri (1987). Theorem 2.1 can then be applied to obtain the asymptotic normality of this linear combination in the case when $\kappa=\infty$. Proposition 3.3 finally follows from Le Cam's third lemma.

The nonsingularity of $\mathbf{W}_{\Psi}^{2}$, hence that of $\underline{\mathbf{W}}_{\Psi}^{2}$, results from the fact that the $\Psi_{t}^{(j)}$ 's are linearly independent solutions of

$$
A(L) B(L) \Psi_{t}=0, \quad t \in \mathbb{Z} .
$$


Proof of Proposition 4.1. Let $\left\{\Theta_{t}^{(1)}, \ldots, \Theta_{t}^{\left(p_{1}+q_{1}\right)}\right\}$ and $\left\{\Psi_{t}^{(1)}, \ldots, \Psi_{t}^{\left(p_{1}+q_{1}\right)}\right\}$ denote two arbitrary fundamental systems for (A3.11). Then

$$
\left(\Theta_{t}^{(1)}, \ldots, \Theta_{t}^{\left.p_{1}+q_{1}\right)}\right)=\left(\Psi_{t}^{(1)}, \ldots, \Psi_{t}^{\left(p_{1}+q_{1}\right)}\right) \mathbf{K}^{\prime}, t \in \mathbb{Z},
$$

where $\mathbf{K}$ is some nonsingular constant matrix of dimension $\left(p_{1}+q_{1}\right) \times\left(p_{1}+\right.$ $\left.q_{1}\right)$. Hence

$$
\mathbf{T}_{\theta ; f}^{(n)}=\left(\begin{array}{cc}
\mathbf{I} & \mathbf{0} \\
\mathbf{0} & \mathbf{K}
\end{array}\right) \mathbf{T}_{\Psi ; f}^{(n)}, \quad \mathbf{W}_{\mathbf{\Theta}}^{2}=\underline{\mathbf{K}} \mathbf{W}_{\Psi}^{2} \underline{\mathbf{K}}^{\prime}
$$

and

$$
\begin{aligned}
\mathbf{T}_{\theta ; f}^{(n) \prime} \underline{\mathbf{W}}_{\boldsymbol{\Theta}}^{-2} \mathbf{T}_{\theta ; f}^{(n)} & =\mathbf{T}_{\Psi ; f}^{(n)}\left(\begin{array}{cc}
\mathbf{I} & \mathbf{0} \\
\mathbf{0} & \mathbf{K}
\end{array}\right)\left(\begin{array}{cc}
\mathbf{I} & \mathbf{0} \\
\mathbf{0} & \mathbf{K}^{-1} \mathbf{W}_{\Psi}^{-2} \underline{\mathbf{K}}^{-1}
\end{array}\right)\left(\begin{array}{cc}
\mathbf{I} & \mathbf{0} \\
\mathbf{0} & \mathbf{K}
\end{array}\right) \mathbf{T}_{\Psi ; f}^{(n)} \\
& =\mathbf{T}_{\Psi ; f}^{(n)} \underline{\mathbf{W}}_{\Psi}^{-2} \mathbf{T}_{\Psi ; f}^{(n)} .
\end{aligned}
$$

Proof of Proposition 4.2. The proof straightforwardly follows from Proposition 4.1. Notice indeed that, on account of (A3.10), letting $\mathbf{k}^{\prime}=$ $\left(\left(a_{1}+b_{1}\right), \ldots,\left(a_{\pi}+b_{\pi}\right), k_{1}, \ldots, k_{p_{1}+q_{1}}\right)$, the asymptotic mean of $n^{1 / 2} \mathbf{T}_{\Psi ; f}^{(n)}$ un$\operatorname{der} K^{(n)}(\mathbf{A}, \mathbf{B} ; \gamma, \delta ; f)$ takes the form

$$
\underline{\mathbf{W}}_{\Psi}^{2} \mathbf{k}\left[\boldsymbol{\sigma}^{2} I(f)\right]^{1 / 2} \text {. }
$$

On the other hand, $\mathbf{k}^{\prime} \underline{\mathbf{W}}_{\Psi}^{2} \mathbf{k}=\|\mathbf{a}+\mathbf{b}\|^{2}$, yielding the noncentrality parameter (4.3).

Proof of Proposition 4.3. We know from Section 3.3 that $\mathbf{T}_{\psi}^{(n)}$ is asymptotically sufficient for testing $H^{(n)}(\mathbf{A}, \mathbf{B} ; \cdot)$ against (3.8). $n^{1 / 2} \mathbf{W}_{\Psi}^{-1} \mathbf{T}_{\Psi ; f}^{(n)}$ is thus also asymptotically sufficient since $\mathbf{W}_{\Psi}^{2}$ is a full-rank covariance matrix.

Under $H^{(n)}(A, B ; \gamma, \delta ; f)$, the asymptotic distribution of $n^{1 / 2} \mathbf{W}_{\Psi}^{-1} \mathbf{T}_{\Psi ; f}^{(n)}$ is normal with mean $\mu(\gamma, \delta)=\underline{\mathbf{W}}_{\Psi} \mathbf{k}\left[\sigma^{2} I(f)\right]^{1 / 2}[(\mathrm{~A} 3.12)]$ and identity covariance matrix.

Let us show that when $(\gamma, \boldsymbol{\delta})$ describes $\mathbb{R}^{p_{2}} \times \mathbb{R}^{q_{2}}, \mathbf{k}$ and thus $\mu(\gamma, \delta)$ describe $\mathbb{R}^{\max \left(p_{1}+q_{2}, p_{2}+q_{1}\right)}$. Any value of $\left(a_{1}, \ldots, a_{p_{2}-p_{1}}\right) \in \mathbb{R}^{p_{2}-p_{1}}$ can be reached by choosing appropriate values of $\gamma_{1}, \ldots, \gamma_{p_{2}-p_{1}}$, and $a_{t}, t \geq p_{2}-p_{1}+1$, can be made equal to any solution of $A(L) \Psi_{t}=0$ by adjusting the remaining $p_{1}$ components $\gamma_{p_{2}-p_{1}+1}, \ldots, \gamma_{p_{2}}$ of $\gamma$. A similar result holds for $\mathbf{b}$ and $\boldsymbol{\delta}$. Furthermore, any solution of $A(L) B(L) \Psi_{t}=0, t \geq \pi+1$, can be decomposed into a solution $\Psi_{t}^{a}$ of $A(L) \Psi_{t}=0$ plus a solution $\Psi_{t}^{b}$ of $B(L) \Psi_{t}=0$. This follows indeed from the fact that $A(z)$ and $B(z)$ have no common root: A fundamental system of $A(L) B(L) \Psi_{t}=0$ can thus be formed by juxtaposing a fundamental system $\left\{\Psi_{t}^{(1)}, \ldots, \Psi_{t}^{\left(p_{1}\right)}\right\}$ of $A(L) \Psi_{t}=0$ with a fundamental system $\left\{\Psi_{t}^{\left(p_{1}+1\right)}, \ldots, \Psi_{t}^{\left(p_{1}+q_{1}\right)}\right\}$ of $B(L) \Psi_{t}=0$.

Now, under the condition $(\gamma, \boldsymbol{\delta}) \in \mathscr{D}(d)$, i.e., $\|\mathbf{a}+\mathbf{b}\|^{2} \geq d^{2}$, the values of $\mu(\gamma, \delta)$ are restricted to those satisfying $\mu^{\prime}(\gamma, \delta) \mu(\gamma, \delta)=\mathbf{k}^{\prime} \underline{\mathbf{W}}_{\Psi}^{2} \mathbf{k} \sigma^{2} I(f) \geq$ $d^{2} \sigma^{2} I(f)$. Thus the family of asymptotic distributions of $n^{1 / 2} \underline{\mathbf{W}}_{\Psi}^{-1} \mathbf{T}_{\Psi ; f}^{(n)}$ under $K^{(n)}\left(\mathbf{A}, \mathbf{B} ; \mathbb{R}^{p_{2}}, \mathbb{R}^{q_{2}} ; f \mid d\right)$ is the set of all normal distributions with mean $\mu$ and identity covariance matrix such that $\mu^{\prime} \mu \geq d^{2} \sigma^{2} I(f)$, whereas under $H^{(n)}(\mathbf{A}, \mathbf{B} ; \cdot)$ 
the asymptotic distribution of $n^{1 / 2} \underline{W}_{\Psi}^{-1} \mathbf{T}_{\Psi ; f}^{(n)}$ is normal with mean $\mathbf{0}$ and identity covariance matrix.

It follows from an invariance argument and the Hunt-Stein theorem [see, e.g., Lehmann (1959), Section 8.4] that the maximin most powerful test for this problem is based on the test statistic $n \mathbf{T}_{\Psi ; f}^{(n)^{\prime}} \mathbf{W}_{\Psi}^{-2} \mathbf{T}_{\Psi ; f}^{(n)}$-which is precisely $Q_{A, B ; f}^{(n) *}$. Because of the asymptotic sufficiency of $\mathbf{T}_{\psi ; f}^{(n)}$, this test is also maximin most powerful for $H^{(n)}(\mathbf{A}, \mathbf{B} ; \cdot)$ against any $K^{(n)}\left(\mathbf{A}, \mathbf{B} ; \mathbb{R}^{p_{2}}, \mathbb{R}^{q_{2}} ; f \mid d\right), d \in \mathbb{R}^{+}$.

Corollary 4.4 gives the particular form of the asymptotically maximin most powerful statistic $Q_{A, B ; f}^{(n) *}$ in the case where $p_{1}=q_{1}=0$ and $\pi=\max \left(p_{2}, q_{2}\right)$. This is the only case where $Q_{A, B ; f}^{(n)^{*}}$ turns out to be a quadratic serial rank statistic in the sense of Hallin, Ingenbleek and Puri (1987).

Acknowledgments. The authors acknowledge with sincere thanks the valuable comments and suggestions of the Editor, the Associate Editor and the referee.

\section{REFERENCES}

ANDERSON, T. W. (1959). On asymptotic distributions of estimates of parameters of stochastic difference equations. Ann. Math. Statist. 30 676-687.

ANDERson, T. W. (1971). The Statistical Analysis of Time Series. Wiley, New York.

BARTELS, R. (1982). The rank version of von Neumann's ratio test for randomness. J. Amer. Statist. Assoc. 77 40-46.

BhatTACHARYY, G. K. (1984). Tests for randomness against trend or serial correlation. In Handbook of Statistics: Nonparametric Methods (P. R. Krishnaiah and P. K. Sen, eds.) 4 89-111. North-Holland, Amsterdam.

Box, G. E. P. and Jenkins, G. M. (1970). Time Series Analysis, forecasting and control. Holden-Day, San Francisco.

Box, G. E. P. and Pierce, D. A. (1970). Distribution of residual autocorrelations in autoregressiveintegrated moving average time series models. J. Amer. Statist. Assoc. 65 1509-1526.

Dufour, J.-M. and HalliN, M. (1987). Tests nonparamétriques optimaux pour le modèle autorégressif d'ordre un. Annales d'Economie et de Statistique 5 411-434.

DuFour, J.-M. and KING, M. L. (1986). Optimal invariant test for the autocorrelation coefficient in linear regressions with autocorrelated errors. Technical Report, Dept. Sciences Economiques, Univ. Montréal.

Dufour, J.-M., LePAGE, Y. and Zeidan, H. (1982). Nonparametric testing for time series: A bibliography. Canad. J. Statist. 10 1-38.

DufouR, J.-M. and RoY, R. (1985). Some robust exact results on sample autocorrelations and tests of randomness. J. Econometrics 29 257-273.

DufouR, J.-M. and RoY, R. (1986). Generalized portmanteau statistics and tests for randomness. Comm. Statist. A-Theory Methods 15 2953-2972.

Evans, M. A. and KING, M. L. (1985). Higher order generalizations of first order autoregressive tests. Comm. Statist. A-Theory Methods 14 2907-2918.

GodFrey, L. G. (1979). Testing the adequacy of a time series model. Biometrika 66 67-72.

HAJEK, J. and ŠIDAK, Z. (1967). Theory of Rank Tests. Academic, New York.

HALliN, M. (1986). Nonstationary $q$-dependent processes and time-varying moving average models: Invertibility properties and the forecasting problem. Adv. in Appl. Prob. 18 170-210.

HALlin, M., INGENBleEK, J.-Fr. and PURI, M. L. (1985). Linear serial rank tests for randomness against ARMA alternatives. Ann. Statist. 13 1156-1181. 
Hallin, M., Ingenbleek, J.-Fr. and PURI, M. L. (1987). Linear and quadratic rank tests for randomness against serial dependence. J. Time Ser. Anal. 8 409-424.

HALlin, M. and MÉlaRd, G. (1987). Rank-based tests for randomness against first order serial dependence. Technical Report, Centre d'Economie Mathématique et d'Econométrie, Univ. Libre de Bruxelles, Brussels.

HAllin, M. and PURI, M. L. (1987). Locally asymptotically optimal tests for randomness. In New Perspectives in Theoretical and Applied Statistics (M. L. Puri, J. P. Vilaplana and W. Wertz, eds.) 87-99. Wiley, New York.

HALlin, M. and PURI, M. L. (1988). Locally asymptotically rank-based procedures for testing ARMA dependence. Proc. Nat. Acad. Sci. U.S.A. To appear.

Hosking, J. R. M. (1978). A unified derivation of the asymptotic distributions of goodness-of-fit statistics for autoregressive time-series models. J. Roy. Statist. Soc. Ser. B 40 341-349.

Hosking, J. R. M. (1980). Lagrange multiplier tests of time series models. J. Roy. Statist. Soc. Ser. $B 42$ 170-181.

Hosking, J. R. M. (1981). Lagrange multiplier tests of multivariate time series models. J. Roy. Statist. Soc. Ser. B 43 219-230.

KING, M. L. and HILLIER, G. H. (1985). Locally best invariant tests of the error covariance matrix of the linear regression model. J. Roy. Statist. Soc. Ser. B 47 98-102.

LE CAM, L. (1960). Locally asymptotically normal families of distributions. Univ. California Publ. Statist. 3 27-98.

LehmanN, E. L. (1959). Testing Statistical Hypotheses. Wiley, New York.

LJUNG, G. M. and Box, G. E. P. (1978). On a measure of lack of fit in time series models. Biometrika 65 297-303.

Miller, K. S. (1968). Linear Difference Equations. Benjamin, New York.

Poskitt, D. S. and Tremayne, A. R. (1980). Testing the specification of a fitted autoregressivemoving average model. Biometrika 67 359-363.

Poskitt, D. S. and Tremayne, A. R. (1982). Diagnostic tests for multiple time series models. Ann. Statist. 10 114-120.

Priestley, M. B. (1981). Spectral Analysis and Time Series. Academic, New York.

DÉPARTEMENT DE Mathematique and

InSTITUT DE STATISTIQUe

C.P. 210

UNIVERSITE LIBRE DE BRUXELlES

1050 BRUXELLES

BELGIUM

\author{
DEPARTMENT OF MATHEMATICS \\ SWAIN Hall East \\ INDIANA UNIVERSITY \\ BLOOMINGTON, INDIANA 47405
}

